\title{
XIX.
}

Mitteilung aus der neurologischen Abteilung des Ambulatoriums der Budapester Bezirkskrankenkasse (Ordinarius: Dozent Dr. Artur v. Sarbó).

\section{Über Tetanie im Anschluss an 78 Fälle.}

\author{
Von \\ Dr. Josef Jacobi, \\ em. klin. Assistenten.
}

(Mit 2 Kurven.)

Manchmal sind es zufällige Entdeckungen, manchmal jahrzehntelang dauernde Forschungen, die immer fortschreitende Vervollkommnung der Untersuchungsapparate, in anderen Fällen wieder die aus reichlichem Material gewonnenen Folgerungen, die uns zur Erkenntnis der Ätiologie, Symptomatologie und richtigen Therapie einer Krankheit führen. - Diese letztere Methode war es eigentlich, auf die man früher die Ätiologie der meisten Krankheiten aufbaute. So z. B., dass den Typhus schlechtes Trinkwasser verursache, dass Pneumonie durch Erkältung entstehe, dass Malaria mit den Sümpfen in Verbindung wäre, waren in tausend und abertausend Fällen gesammelte Beobachtungen, und wenn auch einzelne die Frage aufwarfen, ob nicht vielleicht neben diesen auch anderen Krankheitserregern eine Rolle zufalle, und wenn auch spätere Forschungen die Berechtigung dieses Zweifels erhärtet haben, indem sie in den früher erwähnten Fällen den eigentlichen Krankheitserreger nachwiesen, so ist doch die Richtigkeit dieser Erfahrungen nicht anzuzweifeln.

Vielleicht ist es keine unnütze Bemühung, bei der Tetanie, deren ätiologischen Verhältnisse noch so dunkel sind, eine grössere Anzahl von Fällen sowie solche unter selteneren Umständen aufgetretene zu publizieren, da wir ja nicht wissen können, wo ein und der andere Umstand auftaucht, der zur Klärung ihrer Ätiologie führt, oder der Erforschung den richtigen Weg weist. - Hierauf gründe ich die Berechtigung meiner Publikation, indem ich von Anfang an darauf hinweise, dass ein Teil derselben aus Anhänfung statistischer Daten besteht. Doch fühle ich mich zur Publikation dieser meiner Arbeit auch aus dem Grunde berechtigt, weil ich dadurch - wenn auch in geringem Maße - zur richtigen Beurteilung einer Krankheit, deren 
epidemisches Auftreten von mehreren Seiten betont wurde, beizutragen vermeine, um so eher, als mir aus Ungarn keine Publikation bekannt ist, die eine grössere Anzahl von Tetaniefällen umfasst.

\section{Die Tetanie in Ungarn.}

Die ältere ungarische Literatur ist an Publikationen über Tetanie sehr arm.

Die erste Aufzeichnung über dieses Leiden finden wir in der ungarischen Literatur aus dem Jahre 1872 bei Wittmann, der über 6 Fälle berichtet, welche sich alle auf Kinder beziehen, die sich im Alter zwischen 7 Wochen und 3 Jahren befanden, und von denen bei 2 Verdauungsstörungen, bei 3 rachitische Symptome gefunden wurden. - Die Symptomatologie der Tetanie war jedoch damals noch so wenig bekannt, dass Wittmann bei Aufstellung seiner Diagnose bloss auf die in den Beugemuskeln der Extremitäten auftretenden, sich dem Einflusse des Willens entziehenden schmerzhaften tonischen Krämpfe Rücksicht nahm, sowie darauf, dass ein gewaltsames Strecken der von den Krämpfen befallenen Extremitäten die Schmerzen steigerte. - Als häufige Komplikation erwähnt er den Stimmritzenkrampf.

Ungefähr 10 Jahre lang finde ich hierauf in der ungarischen Literatur keine Erwähnung dieses Leidens. Im Jahre 1881 erschienen 2 Studien von Prof. Purjesz; in welchen bereits der Untersuchungen der Trousseauschen, Erbschen und Chrostekschen Symptome ausführliche Erwähnung geschieht. - In der einen Publikation ist von einem 20 jährigen Kolozsvárer Steinmetz die Rede, bei dem typische Symptome von Tetanie vorhanden waren. - Nachdem in diesem Falle das Auftreten des Leidens mit keinem Umstande einen kausalen Zusammenhang zeigte, so müssen wir denselben unter die idiopathischen Tetanien einreihen, - Der ebendaselbst publizierte 2. Fall bezieht sich auf eine 35 jährige Frau, die im Jahre 1873 an der damals grassierenden Cholera sehr schwer erkrankte und bẹ der am 3. Tage nach Aufhören der asphyktischen Periode sehr heftige Schmerzen in der Gegend der Leber sowie tags darauf starke Gelbsucht auftraten, und am selben Tage hatte Patientin 2 Tetanieanfälle. Wegen Obstipation wurde an diesem Tage der Patientin eine Irrigation verordnet, worauf wenig Kot und ein faustgrosser, verwickelter Klumpen ron Ascaris abging, wonach die Schmerzen in der Gegend der Leber sofort, die Gelbsucht bald darauf verschwanden und sich Tetanieanfälle nicht mehr einstellten. Obwohl in der älteren Literatur öfters während Cholera aufgetretene Tetanie erwähnt wird, bält es doch Verfasser für wahrscheinlicher, dass in diesem Falle die Tetanie mit den Ascariden im Zusammenhang stand.

In einer anderen Publikation berichtet ebenfalls Purjesz über 
ein 16 jähriges Mädchen, das im Monat September 1880 mit Phosphor einen Selbstmordversuch beging. Am 20. März 1881 trank sie neuerdings eine Phosphorlösung. Diesmal griffen die Vergiftungssymptome mehr um sich, am 4. Tage nach der Einnahme traten sowobl in den unteren als auch in den oberen Extremitäten typische Tetaniekrämpfe auf, welche durch mehr als 24 Stunden anhielten. Am folgenden Tage Exitus. - Eine der Tetanie zuzuschreibende Veränderung wurde bei der Sektion nicht gefunden. Der zweite, ebendaselbst publizierte Fall ist teils wegen des hohen Alters des Patienten, teils wegen des hervorragend chronischen Charakters der Krankheit von Interesse. Es handelt sich um einen 80 jährigen Steinmetz, der bereits seit seiner Kindheit zeitweilig an Tetanie litt und deshalb schon zu wiederholten Malen an der Kolozsvárer internen Klinik behandelt wurde. Das Krankheitsbild war sehr ausgesprochen, die Ursache konnte aber nicht nachgewiesen werden.

Velies publizierte im Jahre 1887 folgende 4 Fälle: Der erste betrifft einen in Kémeńd wohnhaften 17 jährigen Schneider, bei dem das Leiden im Januar. 1886 auftrat. - Vorher litt Patient seit drei Jabren an Epilepsie. In diesem Falle war es interessant, dass sich die Parästhesien auf die Haut des ganzen Körpers erstreckten und die Krämpfe ausser in den Extremitäten auch im Musculus orbicularis palpebrae et oris, sowie in den Zungenmuskeln auftraten, weshalb Patient während der Anfälle die Augen nicht öffnen konnte und ihm das Sprechen sehr erschwert war. - Bei diesem Patienten bestand auch seit den ersten krampfartigen Anfällen Doppeltsehen.- Der zweite Fall bezieht sich auf einen 24jährigen, seit sechs Jahren an Epilepsie leidenden, in Budapest wohnenden Schlosser, bei dem sich unter hohem Fieber die Tetanie im Monate Februar einstellte. Der dritte Fall ist der einer Budapester, gleichfalls seit mehreren Jahren an Epilepsie leidenden Schneiderin, der vierte bezieht sich auf eine in Neupest. wohnende 28jährige, im fünften Monat stillende blutarme Frau. Bei ersterer trat im Februar, bei letzterer im Monate Januar die Tetanie aut.

Konrad publizierte im Jahre 1888 zwei Fälle aus Hermanostadt. In einem handelt es sich um eine 34jährige Frau mit häuslicher Beschäftigung, die vor dem Auftreten der Krampfsymptome der Tetanie während eines Jahres hindurch crises gastriquesartige Schmerzanfälle hatte. Ausser der Tetanie war bei der Patientin kein anderes Nervenleiden vorhanden. - Bezüglich einer Magenuntersuchung ist in der Krankheitsgeschichte nichts erwähnt, doch lässt die ausführliche, genaue Beschreibung der bei dieser Patientin beobachteten Symptome darauf schliessen, dass - von den Schmerzen und dem Erbrechen abgesehen - andere Symptome seitens des Magens nicht vorhanden waren. - 
Im anderen Falle beobachtete Konrad das in Rede stehende Leiden bei einem 37jährigen Kanzleibeamten, der mit der Diagnose „encephalitischer Stumpfsinn und Alkoholismus" in die Hermannstädter Irrenanstalt aufgenommen wurde. Bei diesem trat in der Anstalt im Monate Februar kruppöse Pneumonie auf und am Tage ihres Auftretens zeigten sich Tetanieanfälle. Patient starb nach einigen Tagen. Bei der Sektion wurde - von anderem abgesehen - in der Rückenmarkshöhle eine grössere Menge Blut gefunden. Das Rückenmark war hyperämisch, stellenweise mit kapillären Blutungen.

Szegö studierte an dem Material des Budapester Stefanie-Kinderspitale die neben Rachitis vorkommenden nervösen Erscheinungen und fand in 16 Fällen neben dem erwähnten Leiden Tetanie. Ausserdem fand er bei vielen Kindern bloss Facialissymptom, in anderen Fällen bloss das Trousseausche, in einigen wieder die beiden zuletzt erwähnten Symptome neben einander.

Preisach veröffentlicht zwei Fälle aus dem Jahre 1895, deren einer sich auf eine 30 jährige stillende Frau, der andere auf eine Kunstblumenarbeiterin bezieht. Bei ersterer war mässige Struma vorhanden; das Leiden trat bei beiden im Monat Januar auf. Patienten wurden mit Thyreoidintabletten behandelt, worauf die Anfälle seltener wurden, resp. wie aus den Krankheitsgeschichten zu entnehmen ist, in einem Falle nach Aussetzen des Mittels keine Anfälle mehr auftraten, im anderen Falle (bei der mit Struma Behafteten) nach einem anfallfreien Zeitraume von drei Wochen dieselben sich neuerdings, obzwar seltener, einstellten. - Preisach fasst seine Beobachtungen dahin zusammen, dass das Sekret der Schilddrüse den Krankheitserreger der Tetanie nicht vernichte, sondern bloss in solcher Weise auf das Nervensystem wirke, dass es dasselbe gegen den Krankheitserreger unempfindlich mache. -

Sarbó teilt aus demselben Jahre drei Fälle mit, von denen sich zwei auf eine 30 jährige Mutter und deren 3 jähriges Kind beziehen. Im dritten Falle beobachtete er das Leiden bei einem 17jährigen Schuhmacher. Nach Anführung der Krankheitsgeschichten verbreitet er sich ausführlich über die Ätiologie der Krankheit und fasst seine diesbezüglichen Ansichten in Folgendem zusammen: „Bezüglich der Ursache der Tetanie können wir daher sagen, dass wir dieselbe in einem grossen Teil der Fälle in einer allgemeinen Ernährungsstörung suchen müssen, welche entweder durch irgend ein Gift, durch infektiöse Leiden oder Rachitis hervorgerufen wird, oder bei Zuständen, wie das Stillen und die Schwangerschaft, auftritt. - Dem gegenüber gibt es eine Gruppe der Tetanie, die in der Gesamtheit ihrer Symptome an ein infektiöses Leiden denken lässt; ob die Tetanie einen spezifischen Erreger besitze, 
haben die bisherigen Untersuchungen nicht erwiesen und unserer Ansicht nach ist auch das Vorhandensein eines solchen spezifischen Agens nicht zu erwarten."

Scheiber berichtet aus dem Jahre 1902 einen Fall von Tetanie bei einer 23jährigen Frau, bei der während der zweiten Schwangerschaft Parästhesien auftraten, die auch wäbrend der dritten Gravidität und Laktation anhielten, wobei in beiden Händen typische Krampfanfülle auftraten und bei einer solchen Gelegenheit sich dieselben auch auf die Muskeln der Zunge und des Kehlkopfes erstreckten.

Goth beschreibt einen Fall von Tetanie, der. im Jahre 1903 auf der Kolozsvárer gynäkologischen Klinik vorkam. - Bei einer 25 jähr. Frau, die ihr viertes Kind im 4. Monat stillte, musste eine Perinealplastik ausgeführt werden, weshalb das Kind zuerst entwöhnt wurde. Zwei Wochen darauf, als die Milch schon vollständig versiegt war, wurde die Operation in Chloroformnarkose ausgeführt, worauf am folgenden Tage typische Tetanie auftrat, die 14 Tage lang in Anfällen anhielt. Verfasser lässt es unentschieden, ob das Chloroform, das vorhergegangene Stillen oder die Operation die Tetanie auslösten.

Sch eib er stellte $1903 \mathrm{im}$ Budapester königl. Ärzteverein eine 34 jähr. im 6. Monat schwangere Frau ror, bei der die Krämpfe zuerst in den Gesichtsmuskeln auftraten, indem sie sich auf einen grossen Teil derselben erstreckten und zwar angeblich schon einen Monat vor der Konzeption; dazu kamen noch im 4. Monat der Schwangerschaft Krämpfe in den Extremitäten.

Ich publizierte im Jahre 1904 vier Fälle, bei denen in jedem Struma vorhanden war. Von denselben wird an anderer Stelle meiner Arbeit ausfăhrlicher die Rede sein.

Schmiedlechner beobachtete im Jahre 1904 bei einer 44 jähr. Frau Tetanie im 7. Monate der 9. Schwangerschaft. Bei dieser Frau waren schon während der 7 . und 8. Schwangerschaft die Finger erstarrt, sie konnte die Hände nicht gebrauchen, „als ob sie aus Holz gewesen wären". Die Rezidive trat diesmal im Mai auf. Patientin gebar noch im selben Monat, also im 7. Monat ihrer Schwangerschaft, einen lebenden Knaben. Die Tetanieanfälle traten auch im Wochenbett auf. Am 5. Tage post partum stieg die Temperatur auf $40,0^{\circ}$ und die an einem Herzleiden laborierende, sehr schwache Patientin starb. Fine Sektion des Rückenmarks wurde von den Angehörigen nicht gestattet.

Ausser diesen Publikationen ist in der ungarischen Literatur von Tetanie mehrfach auf Grund jener Krankenvorstellungen die Rede, welche in den ungarischen wissenschaftlichen Vereinen erfolgten.

Sarbó stellte im Jahre 1897 zwei Fälle im Budapester Ärzteverein 
vor, welche einen 14 jähr. Schlosser und einen 18 jähr. Setzer betrafen. In seinem die Vorstellung begleitenden Vortrage hob er hervor, dass es den Anschein habe, als ob die Tetanie in Budapest ein häufigeres Leiden zu werden beginne als früher, und bewies dies auch damit, dass an der Laufenauerschen Klinik vom Jahre 1888-1897 bloss 1 Fall vorkam, im letzten Jahre dagegen auf einmal 5. Bei der sich entsponnenen Diskussion stimmten sämtliche, weiterhin namentlich anfgezählten Teilnehmer darin überein, dass im Jahre 1897 bedeutend mehr an Tetanie Erkrankte zur Beobachtung kamen, als früher; bloss Gyurmann behauptete, dass er schon seit längerer Zeit in Ofen jährlich 1-2 Tetaniekranke beobachtete. Bei dieser Gelegenheit erwähnte Jendrassik 2 im selben Jahre beobachtete Tetaniekranke, von denen eine eine stillende Frau war. Auch Donath beobachtete im selben Jahre einen Fall. Kétly sab bei einem 12jährigen Knaben in Verbindung mit Obstipation und im selben Jahre bei einem 2 jährigen Kind im Verlaufe eines Darmkatarrhs Tetanie. Taub behandelte gleichfalls um dieselbe Zeit mehrere an Tetanie leidende Patienten, so zuletzt einen 2 Monate alten Säugling, bei dem dieses Leiden im Verlauf von Darmstörungen auftrat.

Hajós stellte im Jahre 1898 eine 40 jährige, seit 1. Jahr stillende Frau, bei der dieses Leiden vorhanden war, im königl. Ärzteverein vor.

Révész demonstrierte im selben Jahre in der ärztlich-naturwissenschaftlichen Gesellschaft des Biharer Komitates den Fall eines 1.2 jäbrigen Knaben, bei dem im Monat Dezember Kopf- und Ohrenschmerzen sowie Schwindel und auf die linken unteren and oberen Extremitäten sich beschränkende Krämpfe auftraten, während das Sensorium ungetrübt blieb. - Mechanische Reizbarkeit gesteigert. Elektrische Untersuchung wurde nicht vorgenommen. Wegen der fast konstanten Dauer der Krämpfe ergab sich kaum die Gelegenheit zur Untersuchung auf Trousseaus Symptom, doch ist es von Interesse, dass auf Druck auf den Nervus ulnaris die Krämpfe aufhörten. Mit Rücksicht auf den sehr atypischen Verlauf dieses Falles ist es nicht. sicher, ob es sich tatsächlich um Tetanie handelte.

Hajós stellte im Jahre 1899 eine 32 jährige, im 5. Monat stillende, an Tetanie leidende Frau vor. Donath hebt bei dieser Gelegenheit hervor, dass die Tetanie keine so seltene Krankheit mehr in Budapest sei, und erwähnt aus dem vorhergehenden Jahre 2, aus dem laufenden Jahre 1 von ihm behandelten Fall.

Sarbó demonstrierte im Jahre 1900 dieses Leiden bei einer 30 jährigen, seit 5 Monaten stillenden Frau.

Ferenczi stellte im Jahr 1903 eine 36 jährige, ihr 7. Kind im 5. Monat stillende, mit Struma behaftete tetaniekranke Frau vor, bei 
der sich der Kropf während der zweiten Schwangerschaft entwickelt hatte und die auch schon während des Stillens ihres 3. Kindes an diesen Krämpfen litt.

Im folgenden Jahr referiert ebenfalls Ferenczi über neuerliche 5 Fälle, und zwar über eine 37jährige Arbeiterin einer Spodiumfabrik, bei der die Schilddrüse geschrumpft und verkalkt war, und bei welcher die Tetanie während derGravidität auftrat. In einem anderen Fall ist von einer 34 jährigen Frau die Rede, bei der der rechtsseitige Lappen der Schilddrüse vergrössert war. Im dritten Fall beobachtete er bei einer 34 jährigen stillenden Frau gleichfalls neben Vergrösserung des rechtsseitigen Schilddrüsenlappens das in Rede stehende Leiden. Ausserdem demonstrierte er die typischen Symptome dieser Krankheit bei einem an Epilepsie sowie bei einem an Diarrhöen leidenden Patienten. Er ist der Ansicht, dass das Stillen die Tätigkeit der Sehilddrüse in verstärktem Maße in Anspruch nehme, so dass dieselbe nicht imstande wäre, die auf das Nervensystem giftig wirkenden Autotoxine zu neutralisieren. Er hält es für wahrscheinlich, dass in allen Fällen von Tetanie bei Schwangeren und Stillenden, auch in jenen, bei denen Struma nicht nachweisbar ist, die Insuffizienz der Schilddrüse dieses Nervenleiden verursache, und glaubt, dass in jedem Fall Thyreoidinverabreichung zu versuchen sei. - Im selben Jahre wurden die von mir publizierten, früher bereits erwähnten, neben Struma aufgetretenen Fälle von Sarbó vorgeführt.

Sehliesslich demonstrierte Szilágyi im laufenden Jahre in der ärztlich-naturwissenschaftlichen Fachsektion des Siebenbürger Museumvereins den Fall einer an Tetanie leidenden Patientin, die bereits seit 10 Jahren an dieser Krankheit litt.

Wenn wir nun die aufgezählten Angaben, welche, wie ich glaube, sämtliche in der ungarischen literatur veröffentlichten Fälle enthalten, zusammenfassen, so sehen wir, dass in denselben von Tetanie bei 25 Kindern und 45 Erwachsenen die Rede ist. Von einem Teil der Fälle ist, nachdem dieselben bloss im Anschluss an die in wissenschaftlichen Vereinen stattgehabten Diskussionen erwähnt wurden, eine genaue Aufzeichnung nicht vorhanden, und wenn wir andererseits die von Sarbó und mir publizierten Beobachtungen, die unter den im Folgenden zu besprechenden 78 Fällen aufgenommen sind, in $\mathrm{Ab}$ rechnung bringen, so verringert sich obige Ziffer bedeutend, doch ist es trotzdem nicht ohne Interesse, in kurzer Zusammenfassung dieselben zu überblicken. - Besonders über Männer wurden wenig genaue Beobachtungen veröffentlicht, doch gehören diese mit Ausnahme eines einzigen Falles alle in die Groppe der idiopathischen Tetanie. Nach der Beschäftigung verteilen sich dieselben folgenderweise: 2 Stein- 
metze, 1 Schneider, 1 Schlosser, 1 Schuster, 2 Lederarbeiter, 1 Diener, 2 Lehrlinge (?), in einem Fall (Konrad) trat die Krankheit bei einem an encephalitischem Stumpfsinn leidenden alkoholistischen Kanzleibeamten im Anschluss an Pneumonie auf. In allen diesen Fällen wurde die Tetanie in den Winter- und Fräbjahrsmonaten beobachtet. Bezüglich des Ortes des Auftretens muss ich bemerken, dass in der grösseren Mehrzahl der Fälle die Betreffenden in Budapest erkrankten; doch finden sich auch Beobachtungen von anderen Orten, so von Klausenburg, Hermannstadt, sogar auch aus der Provinz. Die Patienten waren alle in jüngeren Jahren, ausgenommen den einen von Purjesz publizierten Fall, der mit seinem Alter von 80 Jahren vielleicht in der ganzen Literatur einzig dasteht. Über Frauen haben wir in 18 Fällen genaue Aufzeichnungen, die sich in folgender Weise verteilen: 5 idiopathische Tetanien, darunter 2 mit häuslicher Beschäftigung, 1 Schneiderin, 1 Blumenmacherin, 1 Arbeiterin. In einem Falle wurde die Tetanie im Anschluss an Phosphorvergiftung beobachtet, in einem anderen im Verlauf von Cholera, jedoch wahrscheinlich im Zusammenhang mit Ascariden. Die übrigen 11 Fälle beziehen sich alle auf Schwangere und Stillende und zwar kamen 3 Fälle während der Gravidität, 8 während der Laktation vor. Das Auftreten des Leidens fiel bei diesen auf folgende Monate: Januar 3, Februar 1, Mai 1, Oktober 1, November 3 Fälle. Es ist noch zu erwähnen, dass bei einex Graviden die Schilddrüse in Schwund begriffen war, bei 3 Stillenden und noch bei einer Frau, die ich zu den idiopathischen Tetanien gezählt habe, eine Vergrösserung der Drüse gefunden wurde.

Ich habe die ungarische Literatur ein wenig ausführlicher behandelt, doch geschah dies mit Absicht aus dem Grunde, weil ich nirgends in der ausländischen literatur eine Erwähnung davon gefunden habe, dass Tetanie in Ungarn vorkomme. Es wird nämlich bezüglich dieses Leidens hervorgehoben, dass dasselbe in einigen Städten und Ländern öfters, an anderen Orten selten, an anderen wieder nur vereinzelt zur Beobachtung käme. Besonders Frankl-Hochwart'1) verwendete viel Mühe und Sorgfalt auf die Zusammenstellung, trotzdem erwähnt er an jener Stelle seiner Arbeit, wo ron tetaniereichen, beziehungsweise -armen Ländern und mehreren deutschen Städten die Rede ist, Ungarn nicht. Obwohl sich, wie ich oben erwähnt habe, in dem Werke Frankl-Hochwarts auch darüber Aufzeichnungen finden, in welchen Ländern dieses Leiden scheinbar spärlich vorkommt (England, Skandinavien, Russland, Italien), so können wir doch die Übergehung Ungarns

1) Frankl-Hochwart, Die Tetanie. Spez. Path. u. Therapie herausgegeben von Prof. Nothnagel. XI. Bd., II. Th., IV. Abth. 
bloss dem Umstande zuschreiben, dass von den aus unserem Vaterlande publizierten Fällen kaum einige auf die Zeit ror dem Jahre 1897 fallen (in welchem Jahre nämlich Frankl-Hochwarts. Arbeit erschien) sowie dem, dass aus dem der Arbeit beigefügten, mit grosser Mühe zusammengestellten umfangreichen Literaturverzeichnis, welches die Literatur der Tetanie seit dem Jahre 1830 umfasst einige frühere ungarische Publikationen gleichfalls fehlen. Gerade deshalb, weil ich nirgends in der Literatur eine Erwähnung. dessen gefunden habe, wie die Verhältnisse diesbezüglich in Ungarn. stehen, habe ich es für notwendig gehalten, die ungarische Literatur in ihrer Gesamtheit aufzunehmen, was zufolge der geringen Zahl der Publikationen jetzt noch leicht auszuführen war. Wenn ich zu den aus der Literatur angeführten Daten noch hinzufüge, dass im neurologischen Ambulatorium der Budapester Bezirkskrankenkasse während. der letzten $8 \%$ Jahre 75 Fälle beobachtet wurden, so ist mit Rücksicht darauf, dass in der Literatur sicherlich nur ein kleiner Bruchteil der Fälle vorkommt, und auch von diesen nur ein Teil von uns beobachtet wurde, es klar, dass Budapest nicht zu den Städten gehört, die wenig Tetaniefälle aufweisen. Die angeführten Daten zeigen anch, dass an anderen Orten Ungarns ebenfalls Tetanie vorkommt (Hermanstadt, Kolozsvár usw.); bezüglich letzterer Stadt kann ich noch bemerken, dass ich während jener 8 Jahre (1895-1902) die ich teils als Hörer, teils später in Stellung an der dortigen internen Klinik verbrachte, selbst mehrfache Tetaniekranke beobachten konnte.

Obwohl ich durchaus nicht in Zweifel ziehen will, dass die Tetanie an manchem Orte in grösserer Anzahl, anderswo wieder nur vereinzelt vorkommt, so muss ich doch hervorheben, dass eine richtige Beurteilung dieser Verhältnisse, sowohl durch literarische Angaben als auch durch Befragen einzelner sehr schwierig ist. So z. B. schreibt Frankl-Hochwart auf Grund der Angabe eines Moskauer Nervenarztes über Russland, dass dieser 7 Jahre lang keinen einzigen Fall gesehen habe, im Jahre 1891 auf einmal mehrere Tetaniekranke beobachtete, und dass um dieselbe Zeit auch an einem anderen Moskauer Krankenhause mehrere Fälle aufgenommen wurden; daraus folgert er, wie es scheint, die Seltenheit der Tetanie in Russland. Und siehe da! Einige Jahre nach Erscheinen des Frankl-Hochwartschen Werkes, im Jahre 1900, publiziert $\operatorname{Voss}^{1}$ ) 49 Fälle von der neurologischen Abteilung Rhybalkins aus St. Petersburg, die in den Jahren 1885

1) Voss, Über Tetanie und myotonische Störungen bei dieser Erkrankung. Monatsschrift für Psychiatrie und Neurologie. Bd. 7. 1900.

Deutsehe Zeitsehrift f. Nervenheilkunde, XXXII. Bd. 
bis 1900 beobachtet wurden und von denen gerade auf das Jahr 1891 keine Beobachtung fällt.

In demselben Werke erwähnt Frankl-Hochwart auch, es sei zweifelhaft, ob in Wien, das an Tetaniefällen so reich sei, früher ebenfalls so viele mit Tetanie behaftete Kranke gewesen wären, als in den letzten 25 Jahren. Eine sichere Antwort lässt sich darauf, wie er meint, nachträglich nicht geben, doch lässt sich darauf schliessen und zwar einerseits daraus, dass er in der Literatur aus den 60 er Jahren sehr wenig Angaben gefunden babe, andererseits aus mündlichen Erkundigungen, insofern von diesem Leiden gut kennende ältere Ärzte der österreichischen Hauptstadt behaupteten, dass es in den früheren Zeiten seltener beobachtet wurde. Zum Beweise dafür, dass diese Annahme nicht jeder Grundlage entbehre, führt er noch an, dass es den Anschein habe, als ob in Paris, wo vom Jahre 1830-1860 sehr viel Tetanie vorkam, dieselbe seither bedeutend abgenommen habe.

Auch mit Bezug auf Ungarn, resp. noch eher auf Budapest, kann die Frage aufgeworfen werden, ob das in Rede stehende Leiden nicht etwa bloss in den letzten Jahren hier in grösserer Zahl aufgetreten ist? Man könnte dabei darauf verweisen, dass die zuletzt erwähnten 78 Fälle sowie der grössere Teil der ans der Literatur geschöpften Angaben aus den letzten 10 Jahren stammen. Dafür sprechen auch die ersten, dieses Lueiden behandelnden ungarischen Publikationen, welche hervorheben, dass diese Krankheitsform bei uns sehr selten wäre; ferner würde diesen Umstand auch jene Diskussion beweisen, die im Jahre $1897 \mathrm{im} \mathrm{kgl.} \mathrm{Ärzteverein} \mathrm{stattfand} \mathrm{und} \mathrm{in} \mathrm{welcher} \mathrm{sich}$ die meisten der daran Teilnehmenden dahin äusserten, dass Tetaniekranke in jener Zeit - und wie wir sehen werden, seither - häufiger beobachtet wurden.

Obwohl wir nun auf Grund allen dessen annehmen müssten, dass besagtes Leiden seit ungefähr 10 Jahren bei uns mehr einheimisch ist als früher, und obwohl diese Annahme durch die bereits früher erwähnten, sich auf Wien und Paris beziehenden Bemerkungen FranklHochwarts unterstützt würde, so ist dies jetzt doch schon schwer zu entscheiden, da auch der Umstand in Rechnung zu ziehen ist, dass in vielen Fällen das Leiden leicht unserer Beachtung entgehen kann. Meinerseits glaube ich, dass die in weitere Kreise erfolgte Verbreitung der genauen Kenntnis der Krankheitssymptome dazu beigetragen haben konnte, die Zahl der Fälle zu vergrössern, und noch viel mehr die Aufstellung der Krankenhilfskassen und-Vereine, wodurch der Arbeiterklasse, in der die Tetanie am häufigsten ist, die spezialärztliche $\mathrm{Be}-$ handlung eher zugänglich gemacht wurde; und nacbdem auf diese Weise immer mehr Fälle in die Hand des Spezialisten kamen, so 
wurde deren Aufmerksamkeit immer mehr auf dieses Leiden gelenkt. - Jedoch kann ich zum Beweise dessen, dass auch schon in den achtziger Jahren die Tetanie nicht gar so selten gewesen sein kann, den Umstand anführen, dass Prof. Purjesz in der vor 20 Jahren erschienenen Ausgabe seines Juehrbuchs der internen Medizin schreibt: "Wir haben selbst die Erfahrung gemacht, dass hauptsächlich in manchem Frühjahr hänfiger Patienten mit diesem Leiden in der Ambulanz erscheinen."

Wenn ich jedoch auch bezüglich der idiopathischen Tetanie, auf welche sich eigentlich Frankl-Hochwarts Bemerkungen beziehen, annehmen wollte, dass dieselbe in den letzten Jahren häufiger geworden sei ( $\mathrm{um}$ so eher, weil ich hinsichtlich dieser doch einige Erklärung für das Häufigerwerden finden könnte, wovon ich an einer anderen Stelle meiner Arbeit sprechen werde), so möchte ich doch kaum glauben, dass die während Laktation und Gravidität auftretenden Tetanien früher nicht in ungefähr ebensolcher Anzahl vorgekommen wären, wie in den letzten 10-15 Jahren, trotzdem von denselben in der ungarischen Literatur erst seit damals die Rede ist.

\section{Die ungarische Literatur der Tetanie.}

1) Ferenczi, Szoptatástól kiváltott tyreogen tetania. Demonstration. Orvosi hetilap 1903. Nr. 50.

2) Derselbe, Tetania esetek. Demonstr. Orv. hetilap 1904. Nr. 9.

3) Góth, Gátképlés utáu fellépett tetania esete. Orvosi hetilap 1903. Nr. 9.

4) Hajos, Tetania typusos esete. Demonstr. Orv. hetilap 1898. Nr. 11.

5) Derselbe, Tetania egy esete. Demonstr. Orv. hetilap 1899. Nr. 49.

6) Jacobi, Struma mellett fellépett tetania esetek. Gyógyàszat 1904. Nr. 22. 23.

7) Derselbe, A tetania oktanához tekintettel a pajzsmirigyre. Festschrift Purjesz. Verlag Hornyanszky 1906.

8) Konrád, Adatok a tetania kortanához. Orv. hetilap 1888. Nr. 39. 40.

9) Purjesz, A tetania ok-és gyogytanához. Orv. hetilap 1881. Nr. 28.

10) Derselbe, A tetaniaról. (Arthrogryposis) Gyogyászat 1881. Nr. 29.

11) Preis ạch, A pajzsmirigy hatása a tetanjára. Orv. hetilap 1895. No. 27.28.

12) Révész, Tetania esetek. Orv. hetilap 1898. No. 31.

13) Sarbó, A tetániáról. Orv. hetilap 1896. Nr. 3. 4. 5.

14) Derselbe, Tetania két esete. Demonstr. Orv. hetilap. 1897. Nr. 15.

15) Derselbe, Tetania esete szoptatò nönél. Demonstr. Orv. hetilap. 1900. Nr. 38.

16) Derselbe, Tetánia harom esete. Demonstr. Orv. hetilap 1904. Nr. 14.

17) Scheiber, A Tetania egy nehéz és rendellenes esetéról. Gyogyászat 1902. No. 22 és Orv. hetilap 1902. No. 7.

18) Derselbe, A tetania egy újabb atypicus esetèròl. Gyogyàszat 1904. Nr. 2 és Orv. hetilap 1903. Nr. 6.

19) Schmidlechner, Tetania, gravidarum. Orv. hetilap 1904. Gynaekolog. Beilage. Nr. 3. 
20) Szègö, Az angolkór ideges jelenségéiról. Magy. Orv. archivum. 1894.

21) Szilagyi, Demonstration. Gyógyàszat. 1905. Nr. 11.

22) Velics, Adatok a tetania ok-éstünettanához. Orv. hetilap 1881. No. 17.

23) Wittmann, Arthrogryposis (önszenri izomzsugor [spasmi idiopathici musculares] gyermekeknél). Orvsi hetilap 1872. Nr. 18-21.

\section{78 Fälle von Tetanie.}

Auf der neurologischen Abteilung des Ambulatoriums der Budapester Bezirkskrankenkasse wurden während der $81 / 2$ Jahre seit Eröffnung des Ambulatoriums am 1. Januar 1897 bis zur zweiten Hälfte 1905 insgesamt 75 Tetaniekranke behandelt. - Die Fälle kamen auf

Tabelle I.

\begin{tabular}{c|c|c|c|c}
\hline Lebensjahr & $\begin{array}{c}\text { Gesamtzahl } \\
\text { der } \\
\text { Fälle }\end{array}$ & Männner & Frauen & $\begin{array}{c}\text { Schwanger } \\
\text { und } \\
\text { Stillende }\end{array}$ \\
\hline \hline $8-9$ & 1 & 1 & - & - \\
$10-11$ & - & - & - & - \\
$12-13$ & 1 & - & 1 & - \\
$14-15$ & 8 & 5 & 3 & - \\
$16-17$ & 12 & 11 & 1 & - \\
$18-19$ & 12 & 11 & 1 & - \\
$20-21$ & 7 & 6 & 1 & - \\
$22-24$ & 4 & 1 & 3 & 8 \\
$25-29$ & 12 & 1 & 11 & 5 \\
$30-34$ & 12 & 3 & 9 & - \\
$35-39$ & 7 & 2 & 5 & - \\
$40-50$ & 2 & 2 & - & 18 \\
$50-60$ & - & - & - & 35 \\
\hline Zusammen & 78 & 43 & & \\
\hline
\end{tabular}

der Abteilung des Dozenten v. Sarbó zur Beobachtung, dem ich für die Überlassung des Materials und der Krankengeschichten auch an dieser Stelle meinen besten Dank ausspreche; ebenso auch für die Überlassung der Krankengeschichten zweier an Tetanie leidender Patienten, die sich während oben erwähnter Zeit in seiner Privatordination eingefunden hatten. Einen während meiner Privatpraxis vorgekommenen Fall eingerechnet, wächst obige Zahl auf insgesamt 78. An der Hand meiner Fälle werde ich mich ausschliesslich mit der Tetanie Erwachsener befassen.

Unter meinen Patienten waren 43 Männer und 35 Frauen, von 
denen bei 18 die Tetanie im Verlaufe von Schwangerschaft oder Laktation auftrat; bei den übrigen Frauen sowie bei einem grossen Teil der männlichen Kranken fanden wir fast ohne Ausnahme idiopathische Tetanie, und so werde ich in den hier folgenden Angaben bloss auf die Schwangeren und Stillenden besonders Rücksicht nehmen. Bezüglich des Geschlechtes möchte ich noch bemerken, dass, die Graviden und Stillenden abgerechnet, noch immer die Frauen im Verhältnissse zu den Männern in grösserer Zahl vertreten waren, als in den meisten derartigen Statistiken.

Das Alter der Kranken zeigt Tabelle I.

Tabelle II.

\begin{tabular}{|c|c|c|c|c|c|c|c|c|c|c|c|c|c|}
\hline Jahre & 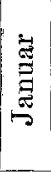 & 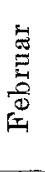 & 胥 & 营 & 留 & 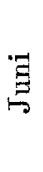 & $\stackrel{-7}{3}$ & $\begin{array}{l}+ \\
\stackrel{2}{0} \\
\stackrel{2}{2} \\
\stackrel{4}{4}\end{array}$ & 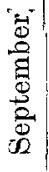 & $\frac{\pi}{0}$ & 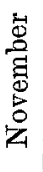 & 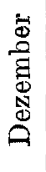 & 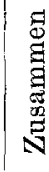 \\
\hline 189 & - & - & 2 & 2 & $1-$ & - & - & - & - & - & - & - & \\
\hline 1898 & - & 1 & - & - & - & - & - & - & - & - & 一 & - & 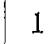 \\
\hline 1899 & - & - & - & 1 & - & - & 一 & - & - & - & - & - & 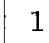 \\
\hline 1900 & 2 & 1 & 1 & 2 & 1 & - & 1 & - & -1 & - & - & - & 8 \\
\hline 1901 & 2 & - & 4 & 1 & 1 & - & 1 & - & - & - & - & - & : \\
\hline 1902 & 5 & 4 & 1. & 2 & 3 & 1 & 1 & - & 1 & 1. & - & 1 & 20 \\
\hline 1903 & 1 & 2 & 7 & 3 & 1 & 1 & - & - & - & - & - & - & 15 \\
\hline 1904 & - & - & 4 & 一 & 2 & 1 & - & 1 & - & - & 1 & 1 & 1 \\
\hline $\begin{array}{r}905 \text { bis } \\
\text { Aug }\end{array}$ & 3 & 1 & 4 & 1 & 1 & - & - & - & & & & & 10 \\
\hline $\begin{array}{l}\text { Säntliche } \\
\text { Fälle }\end{array}$ & 13 & 9 & 23 & 12 & 9 & 3 & 3 & 1 & 1 & 1 & 1 & 2 & 78 \\
\hline 8 & 1 & 2 & 8 & 1 & 2 & 1 & 1 & - & 1 & - & 1 & - & \\
\hline
\end{tabular}

Wie aus dieser Tabelle I ersichtlich ist, wurde bei Männern am häufigsten zwischen dem 14.-22. Jahre, bei Frauen, hauptsächlich weil das Leiden oftmals im Verlaufe der Gravidität und Laktation auftritt, unter den $20-40$ jährigen Tetanie beobachtet.

In den folgenden Tabellen ist das Auftreten der Krankheit bezüglich der Verteilung nach Monaten und Jahren dargestellt und zwar in Tabelle II auf Grund dessen, wann die Kranken sich in der Ordination meldeten, und in Tabelle III, welchen Zeitpunkt dieselben als den des Beginnes ihrer Krankheit angaben. Ein grosser Teil unserex Kranken sucht nämlich mit solchen Leiden, die sie nicht dauernd in 
ihrer Beschäftigung hindern, nicht sogleich ärztliche Hilfe auf, oder wenn doch, so wenden sie sich an den näher wohnenden Bezirksarzt, und manchmal vergehen Wochen und Monate, bis sie, entweder freiwillig oder vom behandelnden Arzte an den Spezialisten gewiesen, erseheinen. Die Zeit des Auftretens der Krankheit ist daher richtiger aus beiden Tabellen zusammengenommen zu bestimmen. Die auf Schwangere und Stillende bezüglichen Daten habe ich in beiden Tabellen in der letzten horizontalen Rubrik gesondert angeführt.

Auf den ersten Blick geht hervor und zwar gleichmässig aus

Tabelle III. $\left.{ }^{1}\right)$

\begin{tabular}{|c|c|c|c|c|c|c|c|c|c|c|c|c|c|}
\hline Jahre & 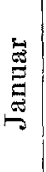 & $\begin{array}{c}0 \\
0 \\
0 \\
0 \\
0 \\
0 \\
0 \\
0\end{array}$ & 胥 & $\frac{7}{2}$ & 总 & $\mid \vec{E}$ & $\stackrel{5}{5}$ & 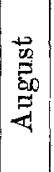 & 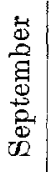 & 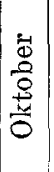 & 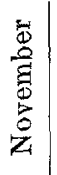 & 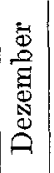 & 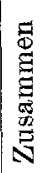 \\
\hline 1897 & - & 1 & 3 & - & - & -1 & - & - & - & - & - & 1 & 5 \\
\hline 1898 & - & - & - & - & - & - & - & - & - & - & - & - & 0 \\
\hline 1899 & - & - & - & 1 & - & - & - & - & - & - & - & 1 & ? \\
\hline 1900 & 2 & - & 1 & 1 & 2 & 1 & - & - & -1 & 1 & - & - & 8 \\
\hline 1901 & - & 2 & 1 & -1 & 1 & 1 & - & - & - & - & 1 & 1. & 7 \\
\hline 1902 & 5 & 2 & 1 & 3 & 3 & - & 1 & -1 & 1 & 1 & - & - & 17 \\
\hline 1903 & 2 & 5 & 2 & -1 & 2 & -1 & - & - & - & - & -1 & - & 11 \\
\hline 1904 & 1 & - & 2 & 1 & 1 & 1 & -1 & $1-$ & - & - & 1 & 2 & 9 \\
\hline $\begin{array}{r}1905 \text { bis } \\
\text { Aug }\end{array}$ & 4 & 2 & 2 & - & - & - & - & - & & & & & 8 \\
\hline $\begin{array}{l}\text { Sämtliche } \\
\text { Fälle }\end{array}$ & 14 & 12 & 12 & 6 & 9 & 3 & 1 & $1-$ & 11 & 2 & 2 & $\check{0}$ & 67 \\
\hline $\mathrm{u}$ & 2 & 3 & 2 & $1 \mid$ & 2 & 1 & 1 & - & -1 & 1 & 2 & -1 & \\
\hline
\end{tabular}

beiden Tabellen, dass die Zahl der Erkrankungen an Tetanie in den ersten fünf Monaten des Jahres am grössten ist, am geringsten dagegen in den Monaten Juli-Oktober. Auch zeigt die Tabelle II, dass die meisten Kranken sich im Monate März meldeten, während die Tabelle III gegen Ende des Jahres eine Steigerung aufweist. Wenn wir die aus beiden Zusammenstellungen gewonnenen Angaben auf ein und derselben Tabelle graphisch dar-

1) In der dritten Tabelle ist die Zahl der Fälle eine kleinere, nachdem der Zeitpunkt des Auftretens der Krankheit nicht in jedem Falle zu bestimmen war. 
stellen, so geben dieselben, sich gegenseitig ergänzend, ungefähr so eine Kurve, die jener sehr ähnlich ist, welche Frankl-Hochwart in seiner Tabelle erhielt, die die Zeit der Aufnahme der während 16 Jahren (1880-1895) am Wiener Allgemeinen Krankenhause behandelten 368 Fälle von Tetanie aufweist (siehe Tab. IV und V.)

Der grösste Teil der Patienten suchte mehrmals die Ordination auf, viele kamen sogar Jahre hindurch mit diesem Leiden. Über einen längeren, sich auf 7-8 Monate erstreckenden interparoxysmalen Zeit-

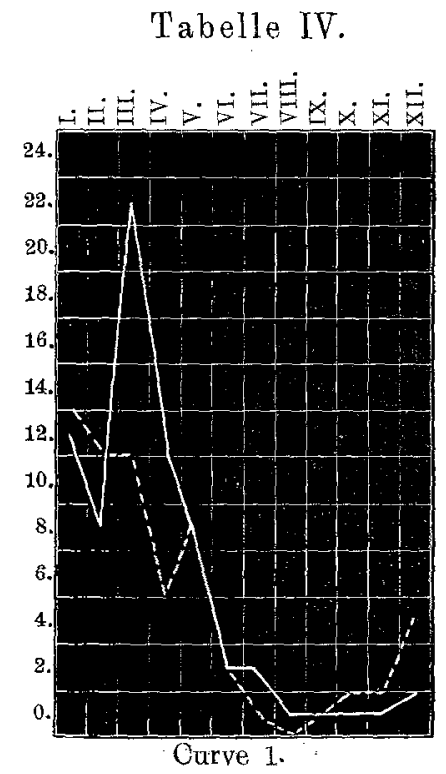

Eigene Fälle.

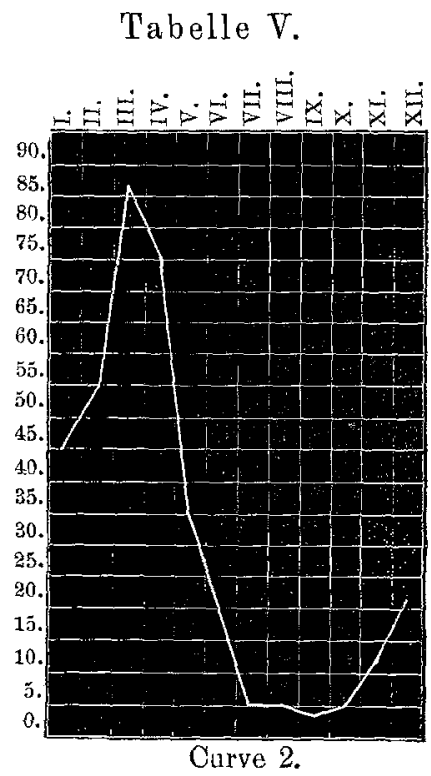

Frankl-Hochwarts Tabelle.

Gemäss ihres Erscheinens in der Ordination.

........ Gemäss des Auftretens der Krankheit.

raum fand ich bei 10 Kranken eine Aufzeichnung und zwar in einem Falle über 5, in fünf Fällen über je 3, in vier Fällen über je 2 solche längere, anfallfreie Perioden. - Das Auftreten neuerlicher Anfälle fiel bei diesen Kranken in die folgenden Monate:

$$
\begin{aligned}
& \text { I. II. III. IV. V. VI. VI. VIII. IX. X. XI. XII. } \\
& \begin{array}{llllllllllll}
3 & 7 & 10 & 2 & 2 & 1 & - & - & 2 & - &
\end{array}
\end{aligned}
$$

Einige Kranke gaben bei ihrem ersten Erscheinen an, dass sie schon vor 1-2 Jahren und zwar immer in der kälteren Jahreszeit, solche Krämpfe hatten. - Wie ersichtlich, ist auch die Rezidive der Krankheit in jenen Monaten eine häufigere, in welchen 
sich dieselbe in grösserer Zahl einzustellen pflegt. Zugleich geht aus dem eben Gesagten hervor, dass bei vielen Patienten das Leiden Jahre hindurch anhielt, jedoch während der Sommermonate sich anch diese besser fühlten. Die Verteilung meiner gesamten Fälle nach Jahren ist aus der letzten vertikalen Rubrik der Tabellen II und III ersichtlich, aus der auch hervorgeht, dass sich in den einzelnen Jahren Tetaniekranke in sehr verschiedener Zahl meldeten. Während z. B. die Jahre 1898 und 1899 bloss mit nur je 1 Fall vertreten sind, kamen in den Jahren 1902 und 1903 - also in solchen Jahren, die die meisten Fälle aufwiesen - 20, resp. 15 solcher Patienten zur Beobachtung.

Bei Beurteilung dieses Umstandes müssen wir vor allem berück-

Tabelle VI.

\begin{tabular}{l|c|c|c}
\hline \multirow{2}{*}{ Jahre } & $\begin{array}{c}\text { Mitglieder- } \\
\text { zahl der } \\
\text { Kranken- } \\
\text { kasse }\end{array}$ & $\begin{array}{c}\text { Anzahl der } \\
\text { Nerven- } \\
\text { kranken }\end{array}$ & $\begin{array}{c}\text { Fälle } \\
\text { von } \\
\text { Tetanie }\end{array}$ \\
\hline 1897 & 52988 & 634 & 4 \\
1898 & 54594 & 794 & 1 \\
1899 & 53855 & 948 & 1 \\
1900 & 50531 & 981 & 8 \\
1901 & 52056 & 1133 & 9 \\
1902 & 55736 & 1216 & 20 \\
1903 & 58968 & 1515 & 15 \\
1904 & 60614 & 1650 & 10 \\
1905 inkl. August & $?$ & 1240 & 10
\end{tabular}

sichtigen, ob nicht vielleicht während dieser Zeit in analoger Weise die Zahl der Mitglieder der Krankenkasse sowie die Zahl derjenigen Kranken, die sich in der Ordination der neurologischen Abteilung meldeten, Schwankungen zeigte. Obenstehende Tabelle VI gibt auf beide Fragen Antwort.

Wie ersichtlich ist, schwankte die Zahl der Mitglieder der Krankenkasse während dieser 9 Jahre verhältnismässig nur um ein Geringes. Dagegen zeigt die Zahl der in der Ordination erschienenen Nervenkranken eine mit den Jahren fortschreitende, zunehmende Steigerung; demnach steht keine Zahlenreihe mit dem Schwanken der Zahl der Tetaniekranken im Verhältnis. Jedoch nicht bloss aus meinen eigenen Fällen, sondern auch auf diesbezügliche statistische Angaben aus der Literatur gestützt, erwäbne ich jene auch von Frankl-Hochwart hervorgehobene Eigentümlichkeit der Tetanie, dass sie in den einzelnen 
Jahren in sehr wechselnder Zahl zu beobachten ist. - Ob dieses Schwanken nach einzelnen Gegenden, nach grösseren Städten gleichmässig ist, diesbezüglich kann ich bloss den einen Vergleich anstellen, den Voss erwähnt. Er berichtet nämlich über 49 Fälle ans St. Petersburg von der neurologischen Abteilung Rybalkins, welche dort während der 16 Jahre von 1885-1900 beobachtet wurden. - Vergleichen wir den auf die Jahre 1885-1895 bezüglichen Teil unserer Angaben mit dem sich auf die entsprechenden 11 Jahre beziehenden Teil von Frankl-Hochwarts ebenfalls auf 16 Jahre sich erstreckender (1880 bis 1895) Statistik, so erhalten wir folgende Reihen:

Jahre: 18851886188718881889189018911892189318941895.

$\begin{array}{llllllllllll}\text { Voss: } & 2 & 11 & 5 & 4 & 4 & 2 & 0 & 1 & 3 & 3 & 2\end{array}$

Frankl-

$\begin{array}{lllllllllll}\text { Hochw.: } 21 & 48 & 32 & 19 & 20 & 23 & 15 & 17 & 13 & 11 & 25\end{array}$

Ist es nicht sonderbar, wie sehr in beiden Reihen das Jahr 1886 hervorragt?

Die Beschäftigung der Patienten geht aus folgenden Angaben hervor:

Männer:

Setzer. . . . . . . . 11 Leichentucharbeiter . . . 1

Schuhmacher . . . . . . 7 Wollfärber . . . . . . . 1

Tischler . . . . . . . . 3 Diener . . . . . . . . 1

Installateure . . . . . 2 Kanzleidiener . . . . . . 1

Arbeiter chem. Fabriken . . 2 Geschäftsdiener . . . . . 1

Tagelöhner . . . . . . . 2 Kellner . . . . . . . . 1

Zeugschmiede . . . . . . 1 Kürschner . . . . . . . 1

Schlosser. . . . . . . . 1 Steinmetz . . . . . . . 1

Maschinenlehrling . . . . 1 Kaufmannslehrling . . . . 1

Bronzearbeiter. . . . . . 1 Schüler . . . . . . . . 1

Geigenmacher . . . . . 1 Farbenpacker . . . . . 1

Frauen:

Schwangere und Stillende:

Mit häuslicher Beschäftigung 3

Kutschersfrau . . . . . . 3

Bürstenbinderin . . . . . 2

Tagelöhnersfran . . . . . 2 Schülerin . . . . . . . 2

Strassenkehrersfrau . . . . 2 Farbenpackerin . . . . . 1

Magazineursfrau . . . . . 1 Wollfärberin . . . . . . 1

Kellermeistersfrau . . . . 1 Schleiferin . . . . . . . 1

Maschinenführersfrau . . . 1 Büglerin . . . . . . . 1

Fabrikarbeitersfrau . . . 1 Hefterin . . . . . . . 1 
Tischlersfrau . . . . . . 1

Schuhmachersfrau . . . . 1

Setzersfrau . . . . . . . 1

Tapezierersgattin . . . . . 1

Kondukteursgattin . . . . 1

Steinbruchsarbeiterin . . . 1

Näherin . . . . . . . . 1
Maschinenmädchen . . . . 1

Wäscherin . . . . . . . 1

Gasglühlichtarbeiterin . . . 1

Eisenbahnbeamtensgattin . . 1

Magazineursgattin . . . . 1

Tagelöhnersgattin . . . . 1

Diese die Beschäftigung der Frauen nachweisende Zusammenstellung gibt zu keiner besonderen Bemerkung Anlass. - Die verschiedenartigsten Beschäftigungen der weiblichen Arbeiterklasse sind darin mit 1-2 Fällen vertreten. Interessanter ist der Ausweis über die Männer. Es ist seit langem bekannt, dass bei gewissen Beschäftigungen die Tetanie häufiger vorkommt als bei anderen, und wir sehen auch aus obiger Zusammenstellung, dass, während die meisten der hier vertretenen Gewerbe bloss mit 1-2 Fällen beteiligt sind, bei gewissen Handwerken dagegen (Setzer, Schuhmacher, Tischler) mehrere Fälle vorkommen. Dass jedoch die Tetanie mit diesen Beschäftigungen in einem - wie immer geringen - Zusammenhang stehe, wären wir nur in dem Falle berechtigt anzunehmen, wenn die vorstehenden Zahlen auch mit der Zahl der diese Gewerbe Betreibenden verglichen, grösser wären, als bei den anderen angeführten Beschäftigungen. In unserem Falle kann die Verteilung der Budapester Bevölkerung nach ihrer Beschäftigung kaum in Betracht kommen, sondern es wird genügen, die diesbezüglichen Verhältnisse bei den Mitgliedern der Krankenkasse ins Auge zu fassen.

In der nachstehenden Tabelle habe ich bloss die männlichen Mitglieder der Krankenkasse berücksichtigt und führe bloss jene Beschäftigungen an, welche unter den männlichen Mitgliedern mit wenigstens 1 Proz. vertreten sind.

Aus der vorstehenden Tabelle, in der sowohl von den Mitgliedern der Krankenkasse als auch von den mit Tetanie Behafteten ungefähr 70 Proz. ihrer Beschäftigung nach dargestellt sind, geht hervor, dass besonders zwei Beschäftigungen unter jenen sind, die eher zu Tetanie inklinieren und zwar das Buchdrucker- und Schusterhandwerk.

Während nämlich von den Mitgliedern der Krankenkasse 6,8 Proz. Buehdrucker sind, finden sich dieselben unter den mit Tetanie Behafteten mit 25,5 Proz. vertreten, also ungefähr $4 \mathrm{mal} \mathrm{mehr,} \mathrm{als} \mathrm{ihrer}$ Anzahl entsprechen würde. Den 3,2 Proz. betragenden Schustern dagegen stehen 16,2 Proz. Tetaniekranke gegenüber, also 5 mal soviel als bei gleichem Verhältnis.

Betrachten wir einmal vergleichsweise einige diesbezügliche $\mathrm{Zu}$ sammenstellungen! 
Frankl-Hochwart fand unter 399 an Tetanie leidenden Männern 174. Schuster, 95 Schneider, 26 Tischler, 20 Schlosser und 19 Drechsler. In Maders ${ }^{1}$ ) Statistik lieferten relativ die meisten Fälle die Schuster, Schneider, Buchbinder und Schlosser. Unter 15 Fällen Hoffmanns ${ }^{2}$ ) sind je 2 Schuster, Schneider, Buchdrucker, andere Beschäftigungen dagegen nur mit je 1 Falle vertreten. Unter 36 männlichen Patienten $\left.\mathrm{Jakschs}^{3}\right)$ sind 15 Schuster, 5 Tischler, 4 Schneider, 3 Drechsler,

Tabelle VII.

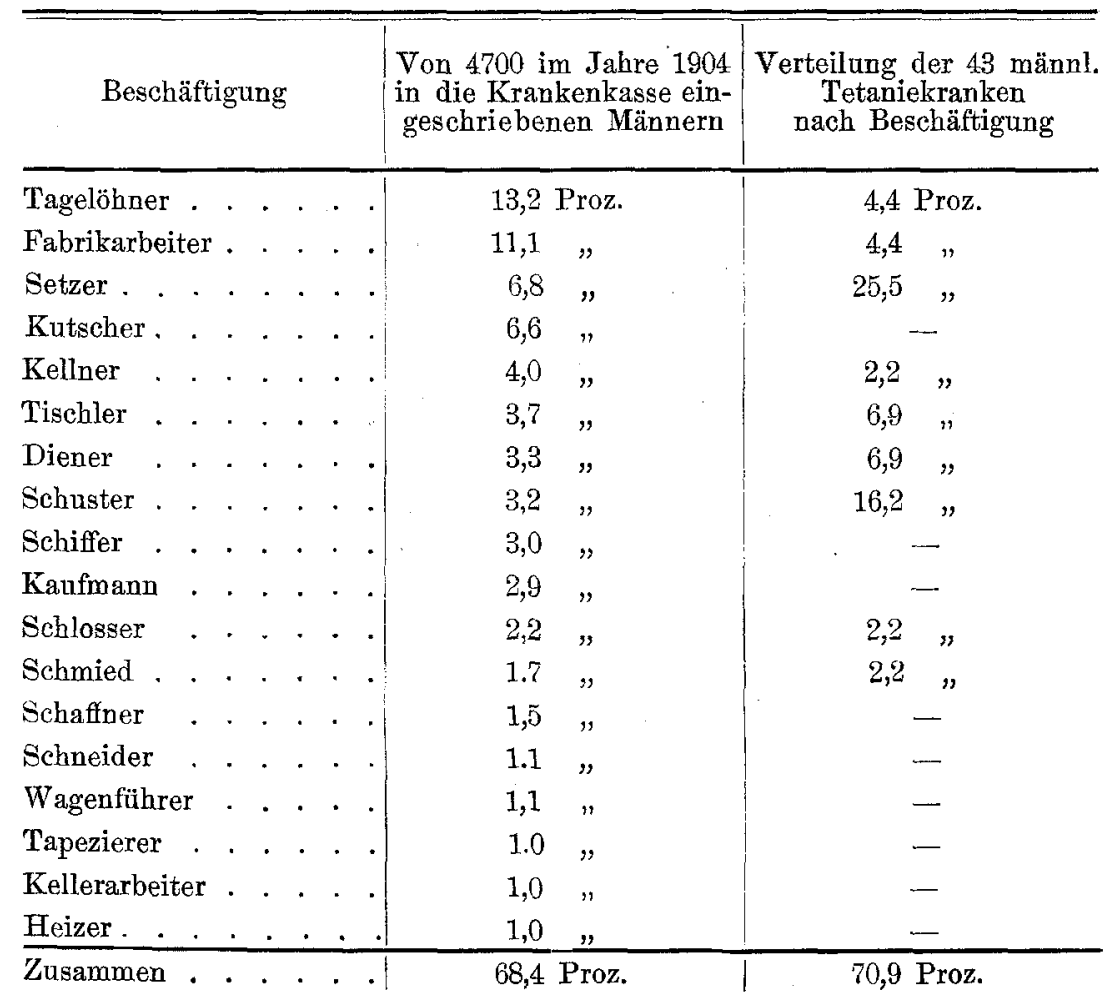

2 Riemer und je 1 Fall mit anderer Beschäftigung. Wenn wir obige, die Beschäftigung meiner Kranken nachweisende Tabelle mit diesen Angaben vergleichen, so finden wir mehrfache Unterschiede zwischen

1) Mader, Ūber die Beziehung der Beschäftigungskrämpfe zur Tetanie. Wiener med. Blätter. 1883. Nr. 16. 17. 20.

2) Hoff man n, Zur Lehre von der Tetanie. Archiv f. klin, Med. Bd. 43. 1838.

3) Jaksch, Klinische Beiträge zur Kenntnis der Tetanie. Zeitschrift f. klin. Med. Bd. 18. Festschrift Leyden. 
denselben: erstens den, dass in jeder Zusammenstellung an erster Stelle die Schuster figurieren, während diese bei uns erst den zweiten Platz einnehmen. Wir haben jedoch gesehen, dass dieser Unterschied nur absolut besteht, relativ dagegen auch bei uns die. Schuster an erster Stelle kommen. Zweitens fällt es auf, dass wir unter unseren Kranken Schneider überhaupt nicht haben; der Grund dafür liegt vielleicht darin, dass, wie wir aus Tabelle VII sahen, verhältnismässig wenig Schneider sich unter den Mitgliedern der Krankenkasse finden, doch erklärt dies den Unterschied nicht vollständig.

Der grösste Unterschied findet sich jedoch darin, dass unter unseren Patienten mit den meisten Fällen die Setzer vertreten sind, also Leute, die sich mit einem solchen Handwerk beschäftigen, bei welchem bisher Tetanie sehr selten in der Literatur erwähnt wurde. Es kann nicht überraschen, dass in einigen Ländern, wo man sich mit Tetanie weniger befasste, weil diese in denselben überhaupt seltener vorkommt, ebenso über Schuster und Schneider, auch von den Setzern nichts erwähnt wird. Jedenfalls ist es aber sehr auffallend, dass wir auch in den aus Wien mitgeteilten Aufzeichnungen dieser Beschäftigung nicht begegnen. Es ist dies um so auffallender, weil die grossen Wiener Statistiken aus den Spitälern stammen, also von Orten, wo Kranke der verschiedensten Beschäftigungen vorkommen. Ich könnte diesen Unterschied nicht anders erklären als damit, dass in Wien bereits zu der Zeit, aus welcher diese Angaben stammen, die Buchdrucker eine eigene Krankenkasse hatten und daher die Spitäler nicht aufsuchten, doch bedarf diese meine Annahme noch der Bekräftigung. Naturgemäss werden wir bei den Buchdruckern dem Blei die Schuld beim Auftreten der Tetanie geben, und wenn wir zur Grundlage unserer Vergleiche die Beschäftigung mit Blei nehmen, so finden wir schon eher einige Ähnlichkeit zwischen den angeführten Statistiken und unseren Angaben. Besonders häufig kommen jedoch Personen, die sich mit Blei beschäftigen, in den Fällen Voss' vor, der dies bei 12 von 39 an Tetanie leidenden Männern nachwies. Dieselben hatten folgende Beschäftigungen: 5 Zimmermaler, 2 Klempner, 2 Schlosser, je 1 Schmied, Kupferarbeiter und Setzer.

Aus dem Gesagten geht hervor, dass die Tetanie in Budapest kein gerade seltenes Leiden ist, sowie dass das grösste Kontingent der Fälle die Monate zwischen Januar und Mai lieferten und dass das Auftreten von Rézidiven ebenfalls in diesen Monaten häufiger ist.

Wir beobachteten das Leiden bei Männern etwas häufiger als bei Frauen; bei letzteren trat das Leiden in grosser Zahl im Verlaufe der Schwangerschaft, resp. Laktation auf. 
Wir sahen es häufiger bei Setzern und Schustern als bei anderen Beschäftigungen.

Im Folgenden will ich nun zur Erwähnung jener bei meinen Kranken beobachteten Symptome übergehen, die nicht zur Symptomatologie der Tetanie gehören. Mit Rücksicht darauf, dass wir von einem Teil derselben aus Erfahrung wissen, dass sie mit dem in Rede stehenden Leiden zusammenhängen, glaube ich dies am übersichtlichsten auf die Art tun zu können, wenn ich meine Fälle nach jenen Gruppen einteile, welche Frankl-Hochwart auf Grund jener Umstände aufgestellt hat, unter denen Tetanie aufzutreten pflegt.

Diese Gruppen sind folgende:

I. Tetanie sonst gesunder Personen (idiopathische, Arbeitertetanie).

II. Tetanie neben Magen- und Darmleiden.

III. Tetanie im Verlaufe von akuten Infektionskrankheiten.

IV. Jene seltenen Fälle der Tetanie, die sich zu solchen Vergiftungen gesellen, welche durch von aussen in den Organismus gebrachte Giftstoffe verursacht werden.

V. Tetanie der Schwangeren, Gebärenden und Stillenden.

VI. Bei Schilddrüsenexstirpation und Schilddrüsenmangel vorkommende Tetaniefälle.

VII. Tetanie in Verbindung mit anderen Nervenleiden.

Diese Gruppierung ist schon vom Gesichtspunkte der leichteren Besprechung sehr gut, dabei entspricht sie - was die Hauptsache ist - unserem heutigen Wissen, nur dürfen wir nicht - worauf bereits Frankl-Hochwart nachdrücklichst aufmerksam gemacht hat diese Umstände mit der Krankheitsursache der Tetanie identifizieren, nachdem wir heutzutage, trotzdem wir sehen, dass die Tetanie mit diesen Umständen zusammenhängt, doch nicht wissen, ob diese direkte Krankheitserreger sind, oder bloss für dieses Leiden die Disposition schaffen.

In einigen Fällen stösst, wie wir sehen werden, diese Gruppierung auf Schwierigkeiten, da ein und die andere Beobachtung auch in zwei verschiedene Gruppen hineinpasst. Besonders ist es fraglich, wo wir die Setzer einteilen sollen, die, wie wir gesehen haben, in unseren Fällen in der Überzahl sind. Bei einem und dem anderen derselben 
konnten wir nämlich auf Grund einiger Symptome an Bleivergiftung denken, bei den meisten jedoch waren solche Symptome nicht vorhanden, weshalb wir diese teils in die Gruppe der sich "an Vergiftungen anschliessenden“, teils unter die „bei sonst gesunden Handwerkern " auftretenden Tetanien einteilen.

Ich werde im Folgenden die erwähnten 7 Gruppen nicht in derselben Reihenfolge behandeln, sondern zuerst jene Fälle auswählen, die leichter zu klassifizieren sind.

1. Tetanie bei Strumaexstirpation sowie bei mangelnder Schilddrüse.

Die in diese Gruppe gehörigen Fälle sind am leichtesten zu klassifizieren, doch befand sich unter meinen Fällen kein solcher. Ich möchte jedoch diese Gruppe in der Weise erweitern, dass ich alle jene Fälle hier einreihe, in denen die Tetanie mit der Schilddrüse zusammenzuhängen scheint. Obwohl nicht zu bezweifeln ist, dass neben Struma auch auf anderer Grundlage Tetanie auftreteten kann; so halte ich doch - wie ich in einer früheren Arbeit darauf hingewiesen habe, es meinerseits für wahrscheinlich, dass in jenen Fällen, in denen das in Rede stehende Leiden neben krankhafter Vergrösserung der Schilddrüse auftritt, dasselbe in den meisten Fällen mit der Erkrankung der Schilddrüse in Verbindung steht. In erster Linie könnte ich dies auf die bei M. Basedowii auftretenden Tetaniefälle beziehen, nachdem ich das mehrfache Zusammentreffen dieser beiden verhältnismässig seltenen Leiden doch nicht als Spiel des Zufalls betrachten kann. Ich selbst habe einen solchen Fall beobachtet, mit dessen Einrechnung ich in jener Arbeit 6 hierher gehörige Fälle angeführt habe. Auf Grund der in meiner selben Arbeit vorgebrachten Beweise scheint es wahrscheinlich, dass die Tetanien bei Schwangerschaft und Laktation ebenfalls in der Schilddrüse ihren Ursprung haben. Dessen ungeachtet werde ich letztere in einer besonderen Gruppe erwähnen, und nachdem bei der früher erwähnten, an M. Basedowii leidenden Patientin sich die Anfälle gleichfalls während der Schwangerschaft einstellten, wird auch darüber an jener Stelle gründlicher die Rede sein. Ich möchte jedoch hier jene in ein und derselben Familie vorgekommenen 3 Fälle erwähnen, bei denen in jedem Struma vorhanden war. Ich hatte diese Fälle, als in vielfacher Hinsicht interessante, bereits für sich allein publiziert und will mich deshalb an dieser Stelle bloss auf eine kurze Erwähnung beschränken. Die Beobachtungen beziehen sich auf eine 37jährige Matter, deren 17 jährigen Sohn und 15 jährige Tochter. Bei der Mutter war Tetanoid (Parästhesie, Chrostek-Symptom, gesteigerte elektrische Reizbarkeit), bei beiden 
Kindern dagegen typische Tetanie vorhanden. Diese Familie wohnte jahrelang in Graz, wo sich die Struma bei allen dreien entwickelte, und eben wegen des raschen Wachstums und der Grösse derselben verliessen sie auf ärztlichen Rat Graz und liessen sich in Budapest nieder. Nach einigen Jahren hierortigen Aufenthalts, während welcher Zeit bei allen Dreien die Struma beträchtlich kleiner geworden war, trat bei dem Sohne Tetanie auf, die seither jährlich in den Winterand Frühjahrsmonaten rezidivierte. Zwei Jahre später trat im Monat März bei dem Mädchen ebenfalls typische Tetanie auf und zur selben Zeit wurden bei der Mutter auf Tetanie hindentende Symptome konstatiert, die schon seit längerer Zeit bestanden. Ich halte es für erwähnenswert, dass in allen 3 Fällen zur Zeit des Auftretens der Tetanie die Struma im Abnehmen begriffen war, und besonders muss ich hervorheben, dass die Tetanie bei allen 3 Familienmitgliedern nicht auf einmal, sondern sich in auf Monate und Jahre erstreckenden Intervallen auftrat, dass also keinesfalls, woran man leicht denken könnte, irgend ein plötzlich wirkender äusserer Umstand gleichzeitig bei 3 Mitgliedern derselben Familie das Leiden ausgelöst hatte. Bezüglich dieser Fälle muss ich noch erwähnen, dass einer derselben - der Sohn - zur Zeit des Auftretens der Tetanie Buchdrucker war, also jenes Handwerk betrieb, dessen Vertreter, wie wir gesehen haben, in der grössten Anzahl in unseren Fällen vorkommen. Dass ich trotz alledem diesem Umstande weniger Bedeutung beimesse, obwohl $\mathrm{Pa}-$ tient seine Beschäftigung durch $31 / 2$ Jahre fortsetzte, hat seinen Grund darin, dass sich auch nach Aufhören dieser Beschäftigung, als er in ein Modewarengeschäft eintrat, auch noch nach 2 Jahren im Frühjahr typische Krämpfe einstellten, andererseits darin, dass bei Mutter and Schwester ebenfalls neben Struma Tetanie vorhanden war.

\section{Tetanie der Graviden und Stillenden. (Nach Abortus und Entwöhnung.)}

Von meinen 35 auf Frauen bezüglichen Beobachtungen gehören 18 in diese Gruppe. - Ausserdem bestand in einem Fall der Verdacht auf Gravidität, insofern bei der betreffenden Patientin seit 7 Wochen die Menses ausgeblieben waren. Diesen Fall rechne ich jedoch nicht hierher, weil, abgesehen davon, dass auf Gravidität bloss Verdacht vorhanden war, Tetanie während Schwangerschaft in der Regel in den späteren Monaten derselben aufzutreten pflegt.

Unter den 18 Fällen kam die Tetanie 3 mal während der Gravidität vor, einmal nach Abortus, bei 13 während der Laktation und 1 mal nach der Entwöhnung. Wir beobachteten sie daher in bedeutend grösserer Zahl bei Stillenden als bei Graviden. Von den 
3 Graviden ist bei 2 die Zeit der Schwangerschaft in der Krankengeschichte nicht verzeichnet, doch wissen wir von ihnen, dass bei beiden noch nach 2 Jahren ohne Dazwischenkunft einer neuen Schwangerschaft sich in grösseren Intervallen Krämpfe einstellten. Der 3. Fall ist interessanter, weshalb ich kurz auf denselben eingehe. Es handelt sich um jene an M. Basedowii leidende Patientin, die ich bereits in der früheren Gruppe erwähnt und deren genaue Krankengeschichte ich schon in einer meiner Arbeiten berichtet habe. Es ist eine 32 jährige Patientin, bei der sich während der 3. Gravidität Struma entwickelte. Langsam kamen dann wechselnde Gemütsstimmung, Handtremor und beschleunigter Puls hinzu, wobei sich über der Struma ein Pulsieren und Schwirren entwickelte. Bezüglich der Augen war ausser einem geringen Exophthalmus kein Symptom wahrzunehmen. Bei dieser Patientin traten im Verlauf von 3 auf einander folgenden (6., 7., 8.) Sehwangerschaften immer gegen deren Mitte, typische Tetanieanfälle auf und hielten bei allen drei Gelegenheiten bis zum Ende der Schwangerschaft an. Das Chvosteksche Symptom sowie die Parästhesien waren bei der Patientin konstant vorhanden.

In einem Falle trat, wie erwähnt, die Tetanie nach Abortus auf. Derselbe war von starker Blutung begleitet und einige Tage nachher traten die Krämpfe auf. Diese Patientin hatte noch 10 Monate später sehr heftige Anfälle mit stundenlang andauernden heftigen Krämpfen. Die Kontraktionen der unteren Extremitäten waren derart schmerzhaft, dass sie sich zu solchen Zeiten nicht vom Bette erheben konnte und deshalb der häuslichen Behandlung überwiesen wurde.

Von jenen 13 Fällen, in welchen die ersten Anfälle des in Rede stehenden Leidens sich während der Laktation einstellten, trat in einem die Tetanie zum ersten Mal während des Stillens des zweiten Kindes: auf, erneuerte sich während des Stillens des dritten Kindes und zeigte sich, nachdem sie sich im 7. Monat der vierten Schwangerschaft wieder eingestellt hatte, auch öfter während des vierten Stillens. Hierauf trat wieder eine Pause ein, jedoch nach der 5. Gravidität, welche im 3. Monat mit Abortus endete, traten die Krämpfe wiederum auf. In zwei anderen Fällen erneverten sich die Krämpfe je 3 mal während des Stillens. Von den übrigen Fällen finde ich Folgendes aufgezeichnet: 1 mal während des dritten, 3 mal während des vierten, 2 mal während des fünften und in einem Falle während des sechsten Stillens trat die Tetanie auf und zwar im 5., 6., 7., 8., 9., und 18. Monat der Laktation. Bei einer und der anderen dieser Patientinnen stellten sich auch noch nach Beendigung der Laktation hier und da Krämpfe ein, weshalb sie öfter das Ambulatorium aufsuchten, 
doch wurden sie in den Sommermonaten nur vereinzelt in der Ordination bemerkt.

In einem Falle traten die Tetanieanfälle einige Tage nach der Entwöhnung auf. Diese Patientin meldete sich im Monat Juni; ihr Kind war damals 1 Jahr alt.

Das Lebensalter der in dieser Gruppe erwähnten 18 Kranken schwankte zwischen 22 und 39 Jahren, wie ich es bereits in der Tabelle I detailliert angeführt habe.

Über die Zeit des Erscheinens der Kranken in der Ordination und des Auftretens der Krankheit war bereits in Tabelle II und III die Rede, aus welchen ersichtlich ist, dass bei Graviden und Stillenden die Tetanie gleichfalls in jenen Monaten am häufigsten ist, in denen das Leiden im allgemeinen am häufigsten beobachtet wird. Den Jahren nach ist die Verteilung dieser Fälle folgende: 19001 Fall; 19013 ; 1902 3; 1903 4; 1904 2; 19055 Fälle.

Über diese Kranken kann ich noch Folgendes bemerken: 3 Stillende gaben mässigen Alkoholgenuss zu, eine andere sagte, dass auch ihre Mutter an Krämpfen leide, doch geht aus der Krankengeschichte nicht hervor, welcher Art diese Krämpfe waren. Bei dem sechsmonatlichen Säugling derselben Patientin war sehr ausgesprochenes Ch hrosteksehes Symptom auslösbar. 2 abortierten je 3 mal. Einer starb 1 Kind, einer anderen 3 Kinder angeblich an Kklampsie. Eine Stillende hatte im Alter von 13 Jahren $4-5$ mal ähnliche Krämpfe gehabt. Eine Patientin hatte gleichzeitig mit der Tetanie Neuralgia supraorbitalis. Bei der an M. Basedowii leidenden Graviden war ein rechtsseitiger Lungenspitzenkatarrh nachweisbar und, wie sie angab, warf sie 3 mal Blut aus. Ausser dieser hatte noch eine Patientin Struma. Auf die Untersuchung der Schildarüse legten wir jedoch bloss bei den zuletzt erschienenen Patienten ein grösseres Gewicht. Jene Frau, bei der Tetanie nach Abortus auftrat, hatte Lymphoma. Bei einer Stillenden wurden im Stuhl zahlreiche Askaridenlarven gefunden; die gegen dieselben eingeleitete Behandlung blieb jedoch anf die Tetaniekrämpfe ohne Wirkung. Schliesslich kann ich bezüglich einer Stillenden noch bemerken, dass bei ihr in geringerem Maße Magensenkung rorhanden war. Stagnation war jedoch nicht nachweisbar.

3. Tetanie im Anschluss an Magen- und Darmleiden.

Aufzeichnungen, die sich auf Magen- und Darmleiden beziehen, sind kaum in den Krankengeschichten zu finden, höchstens ist in 1-2 Fällen von Obstipation die Rede. Bloss eine Beobachtung findet 
sich unter meinen Angaben, in welcher die Tetanie mit einer Affektion des Darmkanals im Zusammenhang $z u$ stehen schien. Diese Aufzeichnung bezieht sich auf einen 18 jährigen Tischler, bei dem seit 2 Monaten Bauchkrämpfe und abwechselnd Diarrhoe und Obstipation vorhanden waren, als er, da er in Händen und Füssen eine Art Erstarrung und Ameisenlaufen verspürte, die Ordination aufsuchte. Er gab an, einige Male in den Händen und Füssen Krämpfe gehabt zu. haben. Bei der Untersuchung wurden Chrosteksches Symptom und gesteigerte elektrische Reizbarkeit beobachtet. Bezüglich des Magens wurde bei Untersuchung mittels der Sonde Stagnation nicht gefunden. Patient stand 3 Wochen in Behandlung und nach einer gegen den Magenkatarrh gerichteten Behandlung blieb sowohl dieser als auch die Tetanie aus. Der Fall ereignete sich im Monat März.

4. Tetanie im Anschluss an akute Infektionskrankheiten.

Im Verlaufe infektiöser Erkrankungen wurde Tetanie verbältnismässig selten beobachtet. Frankl-Hochwart erwähnt in seinem grossen Werke auf Grund der Angaben aus der Literatur ungefähr 17 Typhusfälle, die mit Tetanie kompliziert waren; ausserdem wurde das Leiden selten im Verlaufe von Cholera, Influenza, Pneumonie, Angina tonsillaris, Morbilli und Polyarthritis bemerkt. In der älteren Literatur findet sich auch - wie er schreibt - während Malaria aufgetretene Tetanie erwähnt. Ich selbst hatte an der Kolozsvárer internen Klinik und deren Ambulanz während der in den Jahren um 1900 herum herrsehenden grossen Malariaepidemie Gelegenheit, solche Kranke in grösserer Zahl zu sehen; doch haben wir bei keinem derselben Tetanie wahrgenommen, obwohl in denselben Jahren Tetaniekranke zur Beobachtung kamen. Unter den in der Ordination der Krankenkasse beobachteten Fällen fand sich kein einziger in diese Gruppe gehöriger Patient, doch muss ich jedenfalls hier erwähnen, dass alle diese Fälle von einem ambulanten Krankenmaterial geliefert wurden. Den einen Fall aus meiner Privatpraxis kann ich jedoch hier einfügen. Bei einer jungen Frau traten infolge Myelitis transversa Paraparesis und ausgedehnter, stark eiternder Decubitus auf, die Pyämie und Tod zur Folge hatten. Während der duxch die Pyämie verursachten Fieberperioden traten bei der Patientin bei vollständig ungetrübtem Sensorium bilaterale, typisch tetanieartige, sehr schmerzhafte Krampfanfälle auf. Trousseaus Symptom war sehr ansgeprägt, Chrostek schwach auslösbar. Während des Anfalls steigerten sich durch Druck auf den Sulcus bicipitalis die ohnehin heftigen Schmerzen noch mehr. Elektrische Untersuchung konnte nicht vorgenommen werden. Sektion fand nicht statt. 
5. Tetanie in Verbindung mit anderen Nervenleiden.

Über neue Beobachtungen kann ich an dieser Stelle nicht berichten, höchstens könnte ich hier die eben erwähnte an Myelitis leidende Patientin anführen, bei der im Verlauf einer Pyämie, sowie die unter "Tetanie Schwangerer" eingereibte an M. Basdowii leidende Kranke erwähnen, bei der wäbrend mehrerer aufeinander folgender Schwangerschaften Tetanie auftrat.

6. u. 7. Tetanie bei sonst gesunden Personen und in Verbindung mit Vergiftungen.

Wenn wir die vorher erwähnten Gruppen überblicken, so sehen wir, dass in 3 Fällen (2 Frauen, 1 Mann) die Tetanie böchst wahrscheinlich mit Schilddrüsenerkrankung zusammenhing, in 18 Fällen in Verbindung mit der Maternität anftrat, bei einem Manne im Verlanf eines Darmleidens und bei einer Frau im Verlauf einer während Myelitis aufgetretener Pyämie beobachtet wurde. Es bleiben also noch 55 Fälle übrig, die ich noch nicht erwähnt habe und von denen sich 41 auf Männer und 14 auf Frauen beziehen. Wenn ich die Krankengeschichten dieser Patienten durchgehe, so sehe ich, dass in denselben, abgesehen von den gleich zu erwähnenden Vergiftungssymptomen, nur wenig pathologische Veränderungen erwähnt sind. Einige unter ihnen litten vor Jahren an infektiösen Krankheiten, mehrere waren anämisch, zwei hatten Lungenspitzenkatarrh. Bei einem war vor 1 Jahr virulente Lues, bei einem anderen Mittelohreiterung vorhanden. Ein anderer hatte seit seiner Kindheit Nystagmus. Zwei waren, ebenfalls seit ihrer Kindheit, auf beiden Augen blind. Einer litt an supraorbitaler Neuralgie, ein anderer an Migräneanfällen. Gravidität; Laktation, Schilddrüsenerkrankung, Infektionskrankheit, oder Magen- und Darmstörungen, mit denen die Tetanie in Zusammenhang zu bringen gewesen wäre, haben wir in diesen Fällen nicht gefunden. Bei keinem unserer Patienten waren Symptome akuter Vergiftung zu entdecken, ebensowenig fanden wir schwerere Symptome chronischer Vergiftung, und so verdient bloss erwähnt zu werden, dass bei 5 Patienten auf Alkoholismus und bei 1 auf Bleivergiftung hindeutende Symptome vorhanden waren. Die ersteren 5 Patienten hatten folgende Beschäftigungen: je 1 Setzer, Schuster, Tagelöhner, Geschäftsdiener, Büglerin. Der die Symptome der Bleivergiftung aufweisende Patient war Zeugschmied. Diese Fälle traten in den folgenden Monaten auf: je einer in den Monaten Januar, März, Mai und Angust und zwei im April. Von diesem letzteren Fall abgesehen, in welchem ein ausgesprochener Bleisaum die Diagnose bestätigte, fanden wir noch in mehreren Fällen 
bei Beschäftigung mit Blei Tremor der Hand-, Zungen-, lippenmuskulatur, fibrilläre Zuckungen in den Gesichtsmuskeln, doch war mangels anderer Symptome das Bild der Vergiftung nicht genügend ausgeprägt, um diese Fälle in die Gruppe der sich an Vergiftungen anschliessenden Tetanien einreihen zu können; auch waren im grössten Teil der Fälle in den Krankengeschichten auf Vergiftung deutende Symptome überhaupt nicht verzeichnet.

Wenn man nun in Betracht zieht, dass in den oben erwähnten Fällen die Symptome der Vergiftung keine schweren waren, dass sich Übergangsformen fanden, solche nämlich, bei denen die Symptome nicht genug ausgeprägt waren, um eine Vergiftung anzunehmen, im grösseren Teil der Fälle dagegen Symptome, die einen Schluss auf Vergiftung zuliessen, überhaupt nicht gefunden wurden, jedoch all diese Kranken eben jene Beschäftigung hatten, bei welchen wir unseren Fällen die "Tetanie häufiger sahen, so will ich dieselben nicht gesondert behandeln und erwähne sie deshalb in einer Gruppe.

Was die Beschäftigung, das Alter sowie die Zeit des Auftretens der Krankheit bei den in diese Gruppe eingeteilten 41. Männern und 14 Frauen anbelangt, so ergibt sich dies aus den entsprechenden Tabellen nach Abstrahierung der in den früheren Gruppen erwähnten von selbst und daher ist es überflüssig, nochmals darauf zurückzukommen.

Nur so viel möchte ich noch an dieser. Stelle erwähnen, dass 3 Brüder eines Schriftsetzers (17, 20, 22 Jahre alt) Facialissymptome zeigten. Unter diesen gab der älteste, ein Erzgiesser, bei denen das Chrostek-Symptom am lebhaftesten vorhanden war, an, dass er nach der Arbeit in den Füssen Krämpfe zu haben pflege. Die beiden anderen waren Real- resp. Gewerbeschüler. Ein anderer, ebenfalls Schriftsetzer, behauptete, dass einer seiner Brüder vor Jahren beim Militär dasselbe Leiden hatte.

Im Folgenden werde ich jene bei den Patienten beobachteten Symptome anführen, die wir unter die Symptome der Tetanie rechnen, die jedoch seltener $\mathrm{zu}$ beobachten sind.

Bei einer Stillenden schlossen sich bei einem auf den Plexus brachialis ansgeübten Druck die Finger zur Faust. Bei einer anderen beobachteten wir ebensolche spontane Krämpfe. Ein Tagelöhner bemerkte manchmal bloss am Daumen die schmerzhafte Kontraktion. Bei einer Stillenden waren während der sehr langen Anfälle der 1. u. 2. Finger gestreckt, der 3.-5. eingebogen. Bei dem an Bleivergiftung leidenden Zeugschmied kamen Krämpfe in den Gesichtsmuskeln vor. Ein Diener batte vor den Anfällen epigastrische Schmerzen (Diaphragmakrampf?) 
Der Geigenmacher gab an, dass während der Anfälle sein Mund sich zusammenziehe, dabei seine Sprache behindert wäre und es ihm vor den Augen flimmere. Ein Schriftsetzer sagte, dass sich der Krampf auch manchmal am Halse einstellte, als ob sein Kinn sich ausrenken würde; zu solcher Zeit könne er nicht schlucken, sagte er. Einem anderen Schriftsetzer zittert das Kinn, wenn er die Zähne schliesst (Bleivergiftung oder Tetaniesymptom?). Eine Stillende hatte täglich 3-4 mal Kehlkopfkrampf, wobei sie nur schwer Luft bekam und dieselbe pfeifend einzog. Bei einer anderen Stillenden blieb der Atem aus und sie konnte nicht sprechen. Ebenfalls eine Stillende erwähnte, wenn sie seitwärts schaue, verdrehten sich ihre Augen und sie sehe doppelt. Manchmal blieben ihre Augen stehen, sie könne sie nicht drehen. Schliesslich bemerkte ein Mädchen, dass sich während des Anfalls ibre Augen verdrehten.

In den meisten Krankengeschichten fand ich die Aufzeichnung bezüglich der Untersuchung der Kniee und Achillessehnenreflexe und so erwähne ich auch diese. In 30 Fällen wurden dieselben normal, in 9 Fällen lebhaft und in 3 Fällen sehr lebhaft befunden. Von einigen Kranken ist verzeichnet, dass sie erregt und leicht reizbar waren. Zwei hatten während der Anfälle Schwindel. Endlich fand ich bei 3 Patienten die Bemerkung, dass sie manchmal mit Bewusstlosigkeit verbundene Anfülle hatten. Bei allen 3 waren auspesprochenes Chvostek - und Erb-Symptom vorhanden, während das Trosseausche Symptom bloss bei einem auslösbar war und sich bei den beiden anderen auf Druck des Plexus brachialis bloss Prickeln in den Händen einstellte.

Bloss ein Symptom der Tetanie möchte ich hier noch erwähnen, und dies ist die myotonische Reaktion. Dies für åie Thomsensche Krankheit charakteristische Symptom beschrieb bei der Tetanie zum ersten Male Sarbó im Jahre 1895, indem er Folgendes sagte: „Auf Reizung mit stärkerem faradischen Strom kommt der Triceps in tonische Kontraktion, die auch nach Aufhören des Stroms kurze Zeit anhält und ausgesprochen fühlbar ist." Sarbó spricht bloss vom faradischen Tetanus, nachdem er mit dem galvanischen Strom dies Symptom bei seinem Patienten nicht untersuchte. Hoffmann ${ }^{1}$ ) veröffentlichte im Jahre 1897 die Krankengeschichte eines nach Strumaoperation an Tetanie erkrankten Patienten, bei dem er die myotonische Reaktion beobachtete. Wie aus dem eben Gesagten hervorgeht, schreibt er jedoch irrtümlicherweise äber diese Reaktion: „Sie ist

1) Hoffmann, Weiterer Beitrag zur Lehre von der Tetanie. Deutsche ZZeitschrift f. Nervenheilkde. 1897. 9. Bd. S. 278. 
weder bei der idiopathischen Tetanie noch bei der operativen und experimentellen bis jetzt beobachtet worden." Hoffmann weist bei diesem Patienten nicht bloss bezüglich des faradischen, sondern auch des galvanischen Stroms, wie auch bezüglich des mechanischen Reizes der Muskeln nach, dass die Kontraktionen länger andauerten, als die dieselben auslösenden Einwirkungen.

Wir haben diese Reaktion bei 16 unserer Patienten untersucht und in 6 Fällen gefunden, während sie bei 7 nicht zu entdecken, bei 3 dagegen nicht deutlich war, resp. konnten wir bloss so viel verzeichnen, dass die Erschlaffung der Muskeln nach Aufhören der Wirkung des Stroms lässiger erfolgte, als unter normalen Umständen.

Die Untersuchungen nahmen wir immer sowọl mit galvanischem - als anch mit faradischem Strome vor und zwar gewöhnlich an den Muskeln des Biceps, Triceps sowie des Unterarmes.

\section{Zur Ätiologie der Tetanie.}

Die Krankheitsursache der Tetanie ist bis jetzt noch sehr im Dunkel gehüllt, jedoch bieten die schon vorausgeschickten statistischen Daten und besonders die hierher gehörigen Publikationen der Literatur einen gewissen Anhaltspunkt, in welcher Richtung sich die Forschung zu bewegen hat.

Berücksichtigen wir jedoch vor allem die Angaben der Kranken: Fünf Patienten schrieben ihr Leiden der Einwirkung der Kälte zu, einige bemerkten, dass sie sich bei schlechtem Wetter schlechter fühlten. Bei einem, der gelegentlich eines Ganges auf dem Felde stark durchnässt wurde, trat die Krankheit unmittelbar darnach auf. Mehrere gaben an, von den Krämpfen häufiger während der Arbeit als während der Ruhe ergriffen worden zu sein. Eine Stillende erwähnte, dass sie Krämpfe bekomme, sobald sie ihr Kind auf den Arm nehme. Ein Tischler führte sein Leiden auf Überanstrengung, eine Stillende und ein männlicher Patient dagegen auf Erschrecken zurück. Endlich schrieb ein Patient das Leiden einem "bösen Blick“ zu. So viel ist darüber in den Aufzeichnungen zu finden, doch muss ich bemerken, dass ein Teil der Kranken bezüglich der Ursache ihrer Krankheit nicht befragt wurde.

Trotzdem bei den verschiedenen Leiden die Patienten geneigt sind, die Ursache ihrer Erkrankang der Erkältung oder einer Überanstrengung in ihrem Beruf zuzuschreiben, welche Annahme sehr oft grundlos ist, so dürfen wir doch gerade bei der Tetanie diese Angaben der Patienten nicht unberücksichtigt lassen und zwar, nicht bloss aus dem Grunde, weil beide Umstände von den an dieser Krankheit Leidenden besonders häufig erwähnt werden, sondern noch eher deshalb, weil 
sowohl die Erkältung sowie der Umstand, dass die Krämpfe sich öfters während der Arbeit einstellten, einen gewissen Zusammenhang mit dem bereits Gesagten zeigen.

Beim Zustandekommen der Krankheit ist die Rolle der Kälte und Verkühlung damit in Zusammenhang zu bringen, dass die Tetanie eben in jenen Monaten am häufigsten auftritt, in welchen wir diesen äusseren Einwirkungen am meisten ausgesetzt sind. Jene Selbstbeobachtung: einiger Kranken wiederum, dass sie häufiger während der Arbeit Krampfanfälle bekamen, könnte einigen Zusammenhang damit haben, was sowobl die in den früher erwähnten als auch von anderen mitgeteilten Fällen die Beschäftigung nachweisenden Daten zeigen, dass nämlich diejenigen, deren Beschäftigung ein stärkeres Zusammenschliessen der Finger, das Anfassen kleinerer, feinerer Gegenstände notwendig macht, in grosser Zahl unter den. Tetaniekranken vorkommen. Beide Umstände, nämlich das Durchfrorensein und die Verkühlung, sowie das häufigere Auftreten der Krämpfe während der Beschäftigung finden wir öfters in der Literatur erwähnt. Meinerseits möchte ich jedoch diesen beiden Momenten eine verschiedene Rolle zuweisen. Die bei der Beschäftigung notwendigen Muskeltätigkeiten möchte ich höchstens so weit beteiligen, dass sie bei vorhandener Tetanie manchmal zur Auslösung eines Krampfanfalles beitragen; eine bestimmte ätiologische Wichtigkeit möchte ich jedoch den rheumatischen Einwirkungen zuschreiben. Den Umstand nämlich, dass die Tetanie am häufigsten in den Winter- und Frühjahrsmonaten vorkommt, können wir auf andere Weise als durch die Annahme eines infektiösen Agens oder durch die Wirkung rheumatischer Einflüsse kaum erklären. Denn alle jene Umstände, unter welchen die Tetanie in grösserer Zahl auftritt, wie das Stillen, die Schwangerschaft, Magenleiden usw., kommen ebenso in den übrigen Abschnitten des Jahres vor. Die Beschäftigungen, die bei denselben verwendeten Materialien sind dieselben im Sommer und Winter. Es ist zwar ausser Zweifel, dass wir zufolge des Fortschreitens unserer Wissenschaft heutzutage schon bei viel weniger Leiden als früher genötigt sind bezüglich der Ätiologie rheumatische Einflüsse anzunehmen, trotzdem können wir uns der Annahme ihres Vorhandenseins in der Ätiologie ein oder der anderen Krankheit auch heute nicht verschliessen. Wir finden in der Literatur der Tetanie an mehreren Stellen die Frage aufgeworfen, ob die Krankheit nicht durch ein infektiöses Agens hervorgerufen werde, wofür man als hauptsächliches Beweismoment den Umstand anführt, dảss die selbständig auftretenden (idiopathischen) Tetanjen am häufigsten in den Winter- und Frühjabrsmonaten vorkommen. Dieser Beweis verliert jedoch an Kraft dadurch, dass, wie wir wissen und wie wir auch im Rahmen dieser Arbeit ge- 
sehen haben, die während des Stillens und der Gravidität vorkommenden Tetanien eben dasselbe periodische Auftreten zeigen, ja sogar, wie aus Frankl-Hochwarts Zusammenstellung hervorgeht, die Magentetanien gleichfalls in jenen Monaten häufiger sind. Meinerseits halte ich es auch nicht für wahrscheinlich, dass die Tetanie einen spezifischen infektiösen Erreger habe, und ich möchte meine Ansicht auch dadurch unterstützen, dass auch die nach Strumaoperationen auftretenden Tetanien in den Frähjahrsmonaten zu rezidivieren pflegen. Was das Auftreten der zuletzt erwähnten Tetaniefälle betrifft, so könnte dasselbe, nachdem seine Zeit an die Zeit der Operation gebunden ist, natürlicherweise kaum in Betracht kommen. Die im Anschlusse an Strumaoperationen auftretenden Tetanien stellen sich nämlich gewöhnlich einige Tage nach der Operation ein und so ist beziuglich der Zeit ihres Auftretens in erster Linie der Umstand von Einfluss, in welchen Monaten die meisten dieser Operationen ausgeführt wurden. Doch ist überhaupt auf das Auftreten der Tetanie auch der Umstand von Einfluss, in welcher Weise die Operation ausgeführt wird, wie gross das zurückgelassene Drüsenstück ist, und ob die Nebenschilddrüsen unberührt bleiben. Mit Berücksichtigung aller dieser Umstände erwähne ich doch, welche Verteilung nach den einzelnen Monaten ich bei der Tetania strumipiva gefunden habe. In einer an anderer Stelle erschienenen Arbeit habe ich aus der Literatur 62 Fälle von postoperativer Tetanie gesammelt und habe die bei denselben aufgezeichneten verschiedenen Angaben in einer Tabelle dargestellt. Den Zeitpunkt der Operation sowie des Auftretens der Tetanie fand ich in 47 Fällen pünktlich verzeichnet; unter diesen trat in 44 das in Rede stehende Leiden innerhalb 8 Tagen nach der Operation auf, in drei Fällen stellte es sich später ein.

In diesen 44 Fällen verteilt sich die Zeit des Auftretens des Leidens in folgender Weise auf die einzelnen Monate:

$\begin{array}{cccccccccccc}\text { I. } & \text { II. } & \text { III. } & \text { IV. } & \text { V. } & \text { VI. } & \text { VII. } & \text { VIII. } & \text { IX. } & \text { X. } & \text { XI. } & \text { XII. } \\ 4 & 5 & 6 & 4 & 10 & 4 & 2 & 1 & 1 & 3 & 3 & 1\end{array}$

Wie daraus ersichtlich ist, wurden auch die nach Strumaoperation auftretenden Tetanien in den Winter- und Frühjahrsmonaten in grösserer Anzahl beobachtet, obwohl die meisten Fälle auf den Monat Mai fielen, die wenigsten jedoch auf jene Monate, in welchen auch die in eine andere Gruppe der Tetanie gehörigen Fälle seltener zur Beobachtung kommen. Bloss aus diesen Angaben allein wäre es, wie ich schon erwähnt habe, nicht statthaft, Folgerungen zu ziehen; wenn ich jedoch. hinzunehme, dass auch in diesen Fällen von Tetanie die Rezidiven im Frühjahr sich einzustellen pflegen und jene Tierversuche einiger For- 
scher berücksichtige (Horsley, v. Eiselsberg), aus denen hervorgeht, dass bei Tieren nach Schilddrüsenexstirpation öfters Tetanie auftritt, wenn dieselben der Einwirkung von Kälte ausgesetzt werden, als wenn sie in der Wärme gehalten werden, so kann ich aus all diesem darauf schliessen, dass eine derartige Verteilung der Fälle keine zufällige ist, sondern dass auch diese in grösserer Anzahl sich in jenen Jahreszeiten einstellen, in welchen die übrigen Tetaniefälle auftreten. Ich' kann hier - wenn auch ohne Beweiskraft - erwähnen, nach welcher Zeit sich in jenen drei unter den von mir gesammelten Fällen die Tetanie einstellte, in welchen dieselbe nicht gleich nach der Operation auftrat. In einem ist bloss so viel erwähnt, dass sich die Tetanie im ersten Jahre nach der Operation (dieselbe wurde im August ausgeführt) zeigte. In einem anderen (in welchem die Strumektomie im Juni ausgeführt wurde) trat die Tetanie nach 4 Monaten auf; im dritten dagegen stellten sich die ersten Anfälle (nach der im Oktober ausgeführten Operation) im Monate Februar ein. Wenn wir also bezüglich der Tetania thyreopriva operata annehmen, dass auch bei ihr irgend ein Umstand eine Rolle spielt, infolge dessen sie in den Frähjahrsmonaten beobachtet wird, so ist die früher erwähnte Frage auch darauf auszudehnen, ob ein infektiöses Agens oder die sogenannten rheumatischen Einflüsse dabei mitwirken. Das heisst, es ergibt sich die neue Frage, ob der vollständige, respektive partielle Mangel der Schilddrüse, eventuell deren Entfernung den Organismus zur Tetanie bloss disponiert, bei deren Ausbruch jedoch auch rheumatische Einflüsse mitwirken, oder ob auch ein infektiöses Agens notwendig ist? Bis jetzt haben wir die Fälle von Tetania thyreopriva dem Fehlen der Schilddrüse zugeschrieben und alle Umstände, welche ich zum grossen Teil in meiner oben erwähnten Arbeit zusammengetragen habe, schienen dies zu beweisen. Ich will mich nicht in Widerholungen einlassen und so verweise ich bloss an dieser Stelle auf die dort zusammengefassten allgemein bekannten Dinge. Die Erkenntnis dieses neuen Umstandes macht meiner Ansicht nach die Annahme eines infektiösen Agens nicht notwendig, sondern das Leiden wäre, wie dies auch die erwähnten Tierversuche beweisen, eher auf rheumatische Einflüsse zurückzuführen, nachdem ja auch bei den Tieren, wie wir geseben haben, die Temperaturverhältnisse von Einfluss sind. Gegen das Vorkommen eines solchen Krankheitserregers wäre in diesen Fällen das günstige Resultat der Schilddrüsenbehandlung zu verwerten. Ich habe nämlich in der Literatur 11 solcher Fälle gefunden, in welchen bei Tetania strumipriva Schilddrüse verabreicht wurde. Von diesen starben 2, in den übrigen 9 war die Behandlung erfolgreich, während in anderen Fällen von Tetanie bezüglich der Thyreoidin- 
tabletten nur selten ein günstiges Resultat beobachtet wurde. Gegen das Vorkommen eines derartigen Krankheitserregers in diesen Fällen wäre auch noch anzuführen, dass das Vorhandensein und die normale Funktion der Schilddrüse (ich glaube nämlich.kaum, dass jede Tetanie thyreogenen Ursprungs wäre) nicht vor Tetanie schützt, jedoch auch vor keiner infektiösen Krankheit, wogegen wieder das Fehlen derselben auch zu keiner Infektionskrankheit disponiert. Vielleicht könnte ich auch noch erwähnen, dass, während bei Tieren nach Schilddrüsenexstirpation ebenfalls 'Tetanie auftritt, die idiopathische Tetanie, bei welcher für die Existenz eines derartigen Krankheitserregers die meisten Umstände sprechen, bei Tieren nicht beobachtet wurde, wie mir der Assistent der Veterinärhochschule, Herr Koppán yi mitteilte. Wir können es daber für ansgeschlossen betrachten, dass bei der Tetania strumipriva ein infektiöses Agens mitwirke, und ich möchte die Ursache, dass dieselbe dennoch in den Winter- und Frühjahrsmonaten häufiger auftritt, den sogenannten rheumatischen Einflüssen zuschreiben; bezüglich der übrigen Fälle des in Rede stehenden Leidens bleibt jedoch zufolge verschiedener Umstände, die ich sogleich aufzählen will, der Verdacht aufrecht.

Bevor ich jedoch auf die Würdigung derselben eingehe, drängt sich die Frage auf, ob, nachdem mit Rücksicht darauf, dass die Tetanie in den nach Strumaexstirpation aufgetretenen Fällen - wie wir dies als genügend bewiesen annehmen können - nicht als infektiöses Lieiden zu betrachten ist, ob, wie gesagt, sie in den übrigen Fällen oder in einem Teil derselben als solches, nämlich als infektiöses Leiden zu betrachten wäre? A priori würde ich dies nicht für wahrscheinlich halten, doch können wir es trotzdem nicht als ausgeschlossen betrachten, wenn wir berücksichtigen, welcher Unterschied in den einzelnen Fällen bezüglich der Intensität der Symptome, des Verlanfes und der Daner der Krankheit besteht, ferner, wenn wir bedenken, dass sich auch noch darüber streiten lässt, ob die Tetanie in jedem Fall eine Krankheit für sich oder manchmal bloss eine auf Grund anderer Leiden bestehende Symptomengruppe ist, das heisst, in einigen Fällen ein Morbus sui generis, in anderen bloss ein konsekutiver Symptomenkomplex. Aus dem Folgenden geht hervor, ob wir diese Frage beantworten können.

Betrachten wir nun jene Umstände, welche auf einen infektiösen Ursprung der Tetanie schliessen lassen!

Nach Frankl-Hochwarts Zusammenstellung sind dies folgende: das schon erwähnte periodische Auftreten des Leidens; dessen epidemisches und endemisches Auftreten; 
der Umstand, dass es manchmal im Verlaufe infektiöser Krankheiten anftritt;

die neben Tetanie manchmal zu beobachtende balluzinatorische Verwirrtheit, nachdem diese bei infektiösen Leiden häufig vorkommt;

das im Beginn der Krankheit manchmal beobachtete Fieber mit darauf folgender subnormaler Temperatur:

die in derselben Familie und im selben Hanse beobachten mehrfachen Tetaniefälle;

schliesslich das im Verhältnis zu anderen Jahren häufigere Auftreten in manchen Jahren.

Von allen diesen fallen eigentlich nur die zwei ersten Umstände schwerer ins Gewicht, wäbrend die übrigen nur mehr oder weniger schwache Beweise sind. Ohne Zweifel ist es jedoch begründet, auf Grund allen dessen an ein infektiöses Agens zu denken. Doch auch bei Annahme eines solchen bliebe noch vieles unerklärt, wie dies auch Frankl-Hochwart erwähnt; so unter anderem der Umstand, warum diese Krankheit viel häufiger bei Männern als bei Frauen vorkommt. Warum ist sie unter den in Maternität befindlichen Fraven und Stillenden häufiger, wie auch überhaupt zur Zeit einer erhöhten Funktion der Genitalorgane? Warum erkranken unter den Männern in einem viel grösseren Maße solche, die ein gewisses Handwerk betreiben, wie die Schuster, Schneider, Schriftsetzer usw.? Gegen die Existenz eines spezifischen Krankheitserregers spricht der Umstand, dass die im Anschluss an Strumaexstirpationen auftretenden Tetaniefälle, wie dies die Tierversuche zeigen, gleichfalls bei kälterem Wetter häufiger vorkommen, obwohl wir es für genügend bewiesen erachten, dass selbe nicht infektiöser Natur sind. Sogar die Tetanien der Graviden und Stillenden sind, wie wir es im Folgenden sehen werden, mit grösserer Wahrscheinlichkeit ebenfalls thyreogenen Ursprungs und sind doch in ebendenselben Monaten häufiger, in welchen die idiopathische Tetanie häufiger vorkommt. Gegen das Vorhandensein eines in jedem Tetaniefall tätigen infektiösen Agens scheint auch der Umstand zu sprechen, dass Tetanien Gravider und Stillender auch dort vorkommen, wo, wie es scheint, idiopathische Tetanie zu den Seltenheiten gehört, wie auch der Umstand, dass in den einzelnen Fällen die Symptome sich auf verschiedene therapeutische Eingriffe bessern. Was hingegen die idiopathischen 'Tetanien betrifft, so ist uns, wie wir aus dem Folgenden sehen werden, in manchen Fällen die Ätiologie klar genug, anch ohne Annahme eines infektiösen Agens.

Was nun jene Umstände betrifft, die für das Vorhandensein eines spezifischen Mikroorganismus zu sprechen scheinen, so sind dieselben, wenn auch nicht $\mathrm{zu}$ widerlegen, jedoch genügend zu erklären, ohne 
dass man einen infektiösen Ursprung der Tetanie annehmen müsste. Über die Grände, welche nach meiner Ansicht eine so auffallende Begrenzung der Krankheit auf gewisse Jahreszeiten verursachen, habe ich im Vorbergehenden schon gesprochen. Der andere Umstand, welcher am nachdrücklichsten für den infektiösen Ursprung der Tetanie zu sprechen scheint, ist das epidemisch-endemische Auftreten. Dass die Tetanie auf dem Land, in Dörfern nur sehr selten zur Beobachtung kommt, hat seinen Grund hauptsächlich darin, dass das Leiden dort selten vorkommt; doch kann darauf auch der Umstand von Einfluss sein, dass einesteils in der Provinz weniger Ärzte sind, andererseits die schon in Behandlung kommenden Fälle weniger zur Kenntnis gelangen. Wie wir jedoch gesehen haben, sind uns in Ungarn nicht bloss aus Budapest, sondern auch aus anderen Städten und aus der Provinz Fälle bekannt. Eine andere Frage ist es, warum die Tetanie in der Provinz seltener ist, als in den grösseren Städten. Die Ursache davon kann in so zahllosen Umständen liegen (hygienische Verhältnisse, Beschäftigung, Fabrikarbeit usw.), dass wir vorläufig dieser Frage kaum näher treten können, doch wird dies aus dem Folgenden hervorgehen. Anders verhält sich die Sache mit den in Städten vorkommenden ein epidemisch-endemisches Auftreten zeigenden Tetanien. Gegen die Richtigkeit dieser Beobachtung lassen sich kaum Zweifel erheben, obzwar, wie ich an einer früheren Stelle meiner Arbeit erwähnt habe, die Verhältnisse in dieser Hinsicht auch noch nicht genug geklärt sind. Sind aber vielleicht nicht die Ursachen davon, die an gewissen Orten verwendeten Fabrikmaterialien, verschiedene hygienische Verhältnisse oder Nahrungsmittel? Dass das Leiden in verschiedenen Jahren an einzelnen Orten in so verschiedener Anzahl vorkommt, kann zum Teil vielleicht in den klimatischen Verhältnissen, vielleicht aber in der Verschiedenheit der verwendeten Fabrikstoffe seinen Grund haben. Der Umstand jedoch, dass in den an Tetanie reichen Orten (Wien) in den einzelnen Jahren das Leiden in so verschiedener ZahI beobachtet wurde, ist für den infektiösen Ursprung desselben gleichfalls nicht zu verwerten, nachdem das $\mathrm{Zu}$ - und Abnehmen der Fälle keine so regelmässige Wellenlinie zeigt, wie wir sie bei infektiösen Leiden zu sehen pflegen. Jene sporadisch beobachteten Fälle, wo in einer Familie oder in einer Wohnung gleichzeitig mehrere Tetaniefälle beobachtet wurden, sprechen jedenfalls für eine gemeinsame Krankheitsursache, doch könnte nicht mit demselben Rechte angenommen werden, dass irgend ein anderer schädlicher Einfluss, eine giftige chemische Substanz, eine antihygienische Einwirkung oder irgend eine andere gemeinsame Ursache dieselbe zustande gebracht habe?

Zur Unterstützung der Infektionstheorie führt man auch den Umstand an, dass in manchen Fällen die Tetanie mit Temperaturerhöhung 
auftritt. Wenn wir den von den Anhängern dieser Theorie supponierten Krankheitserreger kennen oder dessen Existenz annehmen würden, so möchten wir auf Grund unserer heutigen Fiebertheorie die Temperaturerhöhung diesem Krankheitserreger, resp. seinen Produkten zuschreiben; jedoch berechtigen diese manchmal beobachteten Temperaturerhöhungen auch im Verein mit den erwähnten anderen Umständen uns noch nicht zur Annahme eines solchen, nachdem ja Temperaturerhöhung nicht bloss bei Infektionsleiden vorkommt und andererseits bei der Tetanie zu den selteneren Symptomen gehört.

Die wenigen nach Infektionskrankheiten beobachteten Tetaniefälle können zwar eine Folge dieser Krankheiten sein, doch lässt es sich nicht behaupten, dass sie nnmittelbar durch den Erreger jener Krankheit, noch weniger aber, dass sie durch einen anderen Mikroorganismus hervorgerufen würden.

Was die im Verlaufe von Tetanie manchmal zu beobachtende halluzinatorische Verwirrtheit betrifft, so ist dieselbe wahrlich kein Beweis für das infektiöse Wesen dieser Krankheit, nachdem wir ja auch nach Vergiftungen, nach Ernährungsstörungen solche Symptome sehen.

Aus dem Gesagten geht herror, dass wir keinen Anhaltspunkt haben, einen in jedem Fall von Tetanie tätigen Mikroorganismus anzunehmen. Ich halte jedoch die Existenz eines solchen auch in den Fällen idiopathischer Tetanie nicht für wahrscheinlich und zwar einerseits aus dem Grunde, weil auch bei Annahme eines solchen Mikroorgasnismus noch vieles unerklärt bliebe, andererseits weil alle jene Umstände oder wenigstens ein grosser Teil derselben, wie das periodische Auftreten des Leidens, das Fieber, die Halluzinationen usw., auch in jenen Fällen von Tetanie, in denen wir weniger Grund haben, an die Mitwirkung eines infektiösen Agens zu denken, ebenso vorhanden sind wie bei den idiopathischen Tetanien. Schliesslich auch noch deshalb, weil wir, wie wir sehen werden, einen Teil der idiopathischen Tetanien erklären können, ohne dass wir genötigt wären, ein infektiöses Agens anzunehmen.

Aus dem Gesagten geht ferner auch hervor, dass es nicht möglich ist, jeden Fall von Tetanie auf ein und denselben Krankheitserreger zurückzuführen, wie anch die Wahrscheinlichkeit, dass auch in ein und demselben Fall nur selten ein Grund zum Zustandekommen des Leidens genügend ist. Meinerseits möchte ich, wie ich dies bei der Besprechung der Ätiologie vorausgeschickt habe, einen entschiedenen Einfluss den rheumatischen Einwirkungen zuschreiben, welche bei den zu Tetanie disponierten oder an latenter Tetanie leidenden Personen den Ausbruch des Leidens hervorrufen. Abgesehen von dieser einheitlichen 
auslösenden Ursache möchte ich jedoch die Disposition oder das $\mathrm{Zu}$ standekommen der vorhandenen latenten Krankheit anderen Ursachen zuschreiben, welche ich im Folgenden in jenen Umständen suchen werde, unter denen wir die Krankheit auftreten sehen.

Diese Umstände sind:

Strumaexstirpation und Schilddrüsenerkrankungen.

Gravidität und Laktation.

Vergiftungen durch von aussen in den Organismus gebrachte Giftstoffe.

Infektionskrankheiten.

Magen- und Darmleiden.

Gewisse Beschäftigunge'n (Buchdrucker, Schuster, Schneider).

Dass zwischen diesen Zuständen, Krankheiten, Beschäftigungen und der Tetanie in den entsprechenden Fällen ein gewisser Zusammeuhang besteht, darüber braucht man heute nicht mehr zu sprechen. Bloss in den wenigen, im Anschluss an Vergiftungen beobachteten Fällen kann bezüglich des Zusammenhangs ein Zweifel bestehen. In den von diesen Fällen handelnden Publikationen kann man auch noch heute lesen, dass die Verfasser an das zufällige Zusammentreffen der beiden Leiden denken. Wir wollen nun sehen, ob und worin der $\mathrm{Zu}$ sammenhang zwischen den erwähnten Zuständen und der Tetanie zu finden ist, und wie weit in den einzelnen Fällen auf Grund positiven Wissens die Verbindungsglieder abzuleiten sind.

In den an Strumaoperation -sich anschliessenden Fällen ist das erste Kettenglied der partielle oder vollständige Mangel der Schilddrüse, oder sagen wir, die mangelhafte Funktion derselben, oder der vollständige Ausfall ihrer Tätigkeit; das letzte Glied dagegen die Tetanie. Von den Zwischengliedern könnten wir bloss eines als bekannt annehmen und zwar die Veränderung im Rückenmark, welche man in einem und dem anderen derartigen Fall bei der Sektion in den Vorderhörnern gefunden hat (Nathan Weiss). Ein grosser Teil jener Forscher, die sich mit dem Studium der pathologischen Veränderungen bei der Tetanie befassen, stimmen schon heutzutage darin überein, dass der Ausgangspunkt der typischen Tetaniesymptome sich im Rückenmark befinde und dieser Annahme entsprechen auch die theoretischen Ableitungen. Unser positives Wissen hört jedoch hier schon auf. Und wodurch diese Veränderungen im Rückenmark verursacht werden, diesbezüglich ist bloss als wahrscheinlichste Hypothese die Thyreoidintheorie acceptiert.

Ungefähr so verhält sich auch die Sache in jenen Tetaniefällen, welche sich im Verlaufe von mit Schilddrüsenerkrankung einhergehenden Leiden, dem Myxödem, M. Basedowii, sowie einfacher Struma einstellen. 
Der Zusammenhang zwischen der lirkrankung der Schilddrüse und der Tetanie lässt sich beweisen, bezüglich des Mechanismus jedoch lässt sich bloss jene Hypothese aufstellen wie bei der früheren Gruppe, mit dem Unterschiede, dass, während dort bloss von dem Funktionsmangel der Schilddrüse die Rede war, wir hier in einigen dieser Fälle von einer veränderten Funktion sprechen.

Wo der Zusammenbang der Tetanie mit der Laktation und Gravidität und mit der Erkrankung der weiblichen Sexualorgane zu suchen wäre, diesbezüglich vermutete man schon lange, dass in dieser Reihe ein Bindeglied die Schilddrüse bilde und zwar zufolge jenes $\mathrm{Zu}$ sammenhanges, welcher einerseits zwischen den Schilddrüsenerkrankungen und der Tetanie, andererseits zwischen den Frkrankungen und der Funktion der weiblichen Sexualorgane und dem krankhaften Wesen der Schilddrüse zu beobachten ist. Die diesbezüglichen Beweise habe ich in einer früheren Arbeit auf Grund statistischer Folgerungen geliefert, die aus der Summierung der einschlägigen literarischen Angaben gewonnen wurden. Ich habe nämlich gefunden, dass unter 43 an Graviditäts- und Laktationstetanie Leidenden bei 13 seitens der Sehilddrüse eine Veränderung vorhanden war, und dass in jenen Fällen, in welchen die Tetanie im Verlaufe mehrerer Schwangerschaften resp. Laktationen auftrat, diese Veränderung seitens der Schilddrüse in einem noch grösseren Prozentsatze - in 12 Fällen 6 mal - zu beobachten war. Ferner dass bei jenen, bei welchen während der Gravidität die Strumaoperation ausgeführt wurde, Tetanie in grösserem Prozentsatze auftrat, als bei jenen, bei welchen Gravidität nicht vorhanden war. Alles dies weist darauf hin, dass die Tetanie der Schwangeren und Stillenden ebenfalls thyreogener Natur ist; über die Rolle der Drüse jedoch gibt es auch in diesen Fällen nur Hypothesen.

Wir haben aus dem Vorhergehenden gesehen, dass gewisse Fälle von Tetanie zweifellos mit der Schilddrüse zusammenhängen, und dass wir in einer anderen, gleichfalls nicht kleinen Gruppe von Fällen auf ẹinen solchen Ursprung begründeten Verdacht haben. Es ist natürlich, dass dies zu jener Frage führen könnte, ob nicht die Ursache einer jeden Tetanie in Schilddrüsenerkrankung zu suchen wäre. Wie verlockend auch die Annahme einer solchen einheitlichen Hypothese wäre, so würde dies bloss zu einer weiteren Kette von Hypothesen führen (dass die Schilddrüse berufen sei, verschiedene Gifte im Organismus zu paralysieren oder gegen dieselben zu immunisieren, l. f. c.), und wir könnten zur Unterstützung dieser Hypothese etwas anderes, als das eben Erwähnte, nicht anführen. Höchstens ein Umstand wäre noch im Auftreten der Tetanie, welcher uns in Versuchung bringen könnte. daran zu denken, und das wäre dieser, dass bedeutend mehr Männer 
an Tetanie erkranken, als Frauen. Mit Rücksicht darauf, dass gewisse Umstände daran denken lassen, dass die Rolle der Schilddrüse bei den beiden Geschlechtern eine verschiedene oder wenigstens ihre Funktion von verschiedener Intensität ist, wäre die Annahme dieser Frage nicht unbegründet; trotzdem lasse anch ich sie fallen und werde das verschiedene Verhältnis im Vorkommen der Tetanie bei beiden Geschlechtern in anderen Gründen suchen.

Am unmittelbarsten erscheint der Zusammenhang in jenen wenigen Fällen, in welchen man Tetanie im Anschluss an akute Vergiftungen beobachtet. Die Zahl der hierher gehörigen Fälle ist sehr gering, doch gestatten in denselben sowohl die klinischen Symptome als auch die anzunebmenden pathologisch-anatomischen Veränderungen die Annahme des unmittelbaren Zusammenhangs. Von jenen Fällen, welche von Frankl-Hochwart in dieser Gruppe erwähnt werden, kann ich nur diejenigen hierher rechnen, die im Verlaufe akuter Vergiftungen auftraten. So erwähnte er einen Fall, in welchem nach Morphium, einen anderen, in welchem nach Spermininjektion, und einen dritten, in welchem nach Chloroformnarkose die Tetanie auftrat. Alle diese sind jedoch bloss sich auf einzelne Fälle beziehende Beobachtungen und haben daher wenig. Beweiskraft. Schon mehr Beobachtungen stehen uns über solche Tetaniefälle zur Verfügung, in welchen sie nach Einführung von Mutterkorn in den Organismus und den aus demselben verfertigten Präparaten auftrat, obzwar freilich dies in der grössten Zahl der Fälle bei solchen Individuen angewendet wurde (Schwangere, Stillende und an verschiedenen Genitalkrankheiten leidende Frauen), bei denen Tetanie auch sonst häufiger vorzukommen pflegt. Trotzdem müssen wir doch in einigen Fällen zufolge des temporären Zusammenhanges zwischen dessen Anwendung resp. Aufnahme in den Organismus und der Tetanie die Symptome des in Rede stehenden Leidens der ausschliesslichen Wirkung dieses Mittels zuschreiben. Jedoch auch die erwähnten Zweifel fallen bei den im Verlaufe von Phosphorvergiftung auftretenden Tetanien weg. Solcher Fälle sind mir 4 bekannt. Purjesz ${ }^{1}$ ) erwähnte zuerst solche Fälle, und er beobachtete 2, Stranszky ${ }^{2}$ ) 1 und Routa ${ }^{3}$ ) 1. Von den Fällen Purjesz ist meines Wissens bloss einer ausführlich publiziert, den ich schon bei Anführung der ungarischen Literatur erwähnt habe.

1) Purjesz, A belgyogyászat tankönyve 1889. Abschnitt Tetanie.

2) Stranszky, Ein Fall von Phosphorvergiftung mit Tetanie. Prager med. Wochenschr. 1897. S. 32.

3) Routa, Tetanie nach Phosphorvergiftung. Prager ned. Wochenschr. 1903. S. 479 . 
Im Falle Stranszkys nahm ein 17 jähriges Mädchen am 23. Februar in selbstmörderischer Absicht eine $0,45 \mathrm{~g}$ Phosphor entsprechende Lösung ein, worauf am nächsten Tage die Lebergegend auf Druck empfindlich wurde. Am 26. Februar zeigte sich Ikterus geringeren Grades, und sie klagte über schwache Krämpfe in den unteren Extremitäten; am 27. stellte sich geringe Temperaturerhöhung ein $(37,7)$ sowie in beiden oberen Extremitäten und in den Gesichtsmuskeln Krämpfe und Ameisenlaufen. Chrostek war damals noch nicht nachzuweisen. Am 29. waren die typischen Symptome der Tetanie ausgeprägt und bestanden in dieser Intensität 4 Tage lang. Ikterus verschwand nach eintägiger Dauer. Patientin fühlt sich immer besser, am 7. März verschwanden auch die Symptome der Tetanie und bloss Trousseau war auf längeren Druck auslösbar.

In dem durch Routa beobachteten Falle trank ein 20 jähriges Mädchen am 5. April die Köpfe von 13 Schachteln Zündhölzer in Wasser. Nach 4 Tagen stellte sich schwacher Ikterus ein. Patientin war sebr stark verfallen, somnolent. Hände in Geburtshelferhandstellung, Chrostek- sowie Trousseau-Symptom auslösbar. In beiden Händen das Gefühl sehr vermindert, so dass man die Finger anbrennen konnte. Am 10. April wurde der Ikterus ausgesprochener, im übrigen der Zustand wie am vorhergehenden Tage. Von da an besserte sich der Zustand, und am 13. April hörten sowohl die Symptome der Vergiftung wie auch der Tetanie auf.

Die ein paar Tage nach Einnahme des Giftes auftretende Tetanie, welche mit Heilung der Vergiftung ebenfalls heilt, würde auch schon in einem einzigen Falle, auch wenn wir denselben nicht erklären könnten, den Verdacht des Zusammenhanges erwecken. Wie wir aber sehen, verfügen wir über 4 solche Beobachtungen und ich könnte bebaupten, dass unter den gesamten Tetaniefällen der kausale Zusammenhang in diesen Fällen am klarsten ist. Wie schon früher erwähnt, müssen wir den pathologisch-anatomischen Sitz der Tetanie im Zentralnervensystem, in erster Linie im Rückenmark vorausetzen, wofür wir auch Beweise haben. So beweisen auch v. Sarbos ${ }^{1}$ ) Versuche, dass der Phosphor in den Nervenzellen des Rückenmarks eine körnige Degeneration hervorruft. Obwohl diese Annahme viel Wahrscheinlichkeit für sich hat, können wir es doch nicht für ausgeschlossen halten, dass die Tetanie in diesen Fällen mit Gallenresorption in Zusammenhang steht, und zwar um so weniger, als in einigen Fällen auch bei

1) Sarbo, A házinyúl gerinezveleje ép idegsejtjeinek szerkezete és azok elváltozásai phosphor és morphium mérgezéseknél. Magy. orv. Arch. 1892.

Dentsche Zeitschrift f. Nervenheilkande. XXXII. Bd. 
anderen mit Gelbsucht verbundenen Krankbeiten Tetanie beobachtet wúrde.

Bevor ich weitergehe, kann ich es nicht unerwähnt lassen, dass von obigen, infolge von Phosphorvergiftung aufgetretenen 3 Tetaniefällen der eine im Monat Februar, der andere im März, der dritte im April beobachtet wurde.

Was nun die chronischen Vergiftungen anbelangt, so ist es natürlicb, dass in den neben solchen beobachteten Fällen mit Rücksicht auf die lange Dauer der Einwirkung des Giftes ein zufälliges Zusammentreffen beider Krankheiten leicht möglich ist. Wenn wir jedoch bedenken, dass man Tetanie eben bei Vergiftungen mit solchen Stoffen beobachtet hat, von denen nachgewiesen ist, dass sie so, wie z. B. der Phosphor, das Zentralnervensystem angreifen (Schaffer bezüglich des Bleies), ist es nicht zunächst liegend, dass wir das Zustandekommen der Tetanie diesen zuschreiben? Unter diesen Stoffen ist es hauptsächlich das Blei, dem wir bei Tetaniekranken häufig begegnen, und einige einschlägige Beobachtungen finden sich auch unter meinen oben erwähnten Fällen. Während jedoch neben den Symptomen von Bleigiftung verhältnismässig selten Tetanie beobachtet wurde, finden sich in der neueren Literatur eine beträchtliche Anzahl Beobachtungen, aus denen hervorgeht, dass Tetanie bei Personen vorkommt, die sich mit bleihaltigen Stoffen beschäftigen. Es ergiebt sich daher die Frage, ob in jenen Fällen, in welchen die von Tetanie Befallenen der Möglichkeit einer Bleivergiftung ausgesetzt waren, deren bekannte Symptome jedoch nicht nachgewiesen werden konnten, wir für die Tetanie das Blei verantwortlich machen können. Eine sichere Antwort lässt sich auf diese Frage derzeit nicht geben, jedoch kann ich auf Grund der in vorliegender Arbeit publizierten Fälle aus jenem Umstand darauf schliessen, dass, wie wir gesehen haben, unter 43 Männern 11 Schriftsetzer waren, also eine solche Beschäftigung trieben, bei welcher Bleivergiftung am häufigsten aufzutreten pflegt. Wir können jedoch nicht bloss bei Schriftsetzern, sondern auch bei anderen Beschäftigungen das Blei als Krankheitserreger verdächtigen. So würde ich z. B. in meinen Fällen bei den zwei Monteuren, bei dem Zeugschmied, bei dem Schlosser und bei dem Maschinenlebrling ebenfalls an das Blei als Krankheitserrger denken, und zwar um so eher, weil wir diese Beschäftigungen in den meisten Statistiken finden.

Ich habe eigentlich von den im Anschluss an Vergiftungen auftretenden Tetanien zu sprechen begonnen, doch führten uns diese, wie wir sehen, zu den idiopathischen Tetanien, und so wollen wir jetzt schon bei diesen bleiben. Wie wir wissen, beobachten wir idiopathische Tetanie bei gewissen Beschäftigungen in grösserem Maßstabe als unter 
Leuten, die andere Handwerke betreiben, und besonders auffallend ist es, dass unter den Ackerbautreibenden sowie unter den sich mit geistiger A rbeit Beschäftigenden das Leiden nur sehr vereinzelt vorkommt. Unter Zugrundenahme dieses Umstandes müssen wir bei der idiopathischen Tetanie eigentlich an eine Gewerbeerkrankung denken. Wo ist jedoch der Zusammenhang zwischen den Handwerken und der Tetanie, resp. zwischen der Tetanie und jenen Handwerksarten, bei welchen wir das in Rede stehende Leiden öfters auftreten sehen? Meinerseits möchte ich die Antwort in der durch $O p$ penheim aufgeworfenen Frage suchen: "ob nicht die Ursache der Tetanie in jenen Arbeitsmaterialien zu suchen wäre, welche an den einzelnen Orten von den Handwerkern benutzt werden?" Besonders sind es die Schuster, Schneider, ferner die Buchdrucker und andere sich mit solchen bleihaltigen Materialien Befassende, sowie die Tischler, welche eine grössere Zahl von Tetaniefällen liefern. Bei einer Gruppe derselben haben wir, glaube ich, nicht unbegründeterweise die Ursache in der Bleiresorption gesucht. Welche Stoffe bei Schustern und Schneidern die Tetanie verursachen könnten, darauf könnte ich um so weniger Antwort geben, als ich mit der Technik der Leder- und Tuchfabrikation nicht vertraut bin, doch möchte ich mir erlauben, dem Verdachte Ausdruck zu geben, dass vielleicht in den Materialien, die dort beim Färben zur Verwendung kommen, sich irgend ein solcher chemischer Stoff befindet, und zwar habe ich um so eher darauf Verdacht, weil dieser sowohl beim Leder als auch bei den Stoffen wesentlich derselbe sein kann. Vielleicht sind die mineralischen Farben, welche man noch heutzutage sowohl in der Stoff- als anch Lederfärberei benutzt und die bekanntlich giftige Stoffe, besonders Bleiverbindungen enthalten, Ursache der Tetanie? Vielleicht wäre auch für den Tischler ebenfalls in der Farbe jener schädliche chemische Stoff zu suchen, welcher die Tetanie verursacht.

In meinen eigenen Fällen glaube ich noch bei folgenden die Tetanie auf die Beschäftigung mit schädlichen chemischen Stoffen zurückführen zu können: bei den Arbeitern in chemischen Fabriken, bei Wollfärbern (Blei), bei Bronzearbeitern (Quecksilber), Farbenpackern (Blei), Bürstenbindern (Blei), sowie bei den Arbeitern der Glühstrumpffabriken (Quecksilber); anf die bei letzterer Beschäftigung auftretende Quecksilbervergiftung hat Donath ${ }^{1}$ ) aufmersam gemacht.

Es kann nicht meine Aufgabe sein, mich über alle jene Besehäftigungen, bei denen Tetanie schon beobachtet wurde, auszulassen und in denselben nach der Ursache des Leidens zu forschen, und ich glaube auch nicht, dass in jedem einzelnen selbständig scheinenden

1) Donath Gyula, Orvosi hetilap 1894. Nr. 7. 
(idiopathischen) Fall von Tetanie eine Vergiftung vorliegt. Bloss bezüglich jener Beschäftigungen, bei welchen das Leiden häufiger beobachtet wird, sowie bezüglich ein und des anderen im Verlaufe meiner Fälle vorgekommenen Gewerbes habe ich darauf verwiesen, in welcher Richtung meiner Meinung nach die weitere Forschung auszudehnen wäre, nachdem hierzu mehrere Umstände, die ich hier aufzählen werde, berechtigen. Wir finden das Leiden hanptsächlich bei Leuten mit solcher Beschäftigung, bei denen die Gelegenheit zu einer solchen Vergiftung vorhanden ist; dies würde am besten erklären, warum es mit Vorliebe bei gewissen Beschäftigungen auftritt, sowie auch, warum das Leiden viel häufiger bei Männern als bei Frauen vorkommt, nachdem wir es eben bei jenen Gewerben am häufigsten finden, mit denen sich Framen nur wenig beschäftigen. Unter den hier in Betracht kommenden Gewerben ist es noch am ehesten das Schneidergewerbe, welches auch Frauen betreiben, aber gibt es nicht viel mehr männliche als weibliche Schneider? Mit der Herrenschneiderei beschäftigen sich doch ausschliesslich Männer, mit der Damenschneiderei dagegen zum grossen Teil Männer. Diese Annahme würde auch erklären, warum an einzelnen Orten bei gewissen Beschäftigungen das Leiden häufiger ist, nachdem ja die Leder- und Stofffärberei nicht überall und zu jeder Zeit mit denselben Materialien geschieht; ebenso würde diese Annahme auch darauf eine Antwort geben, warum das Leiden sich nicht bei denjenigen einzustellen pflegt, die sich mit Ackerbau und geistiger Arbeit befassen, und warum diese Krankheitsform von den Jahren der Kindertetanie angefangen bis zum 14-15 Jahre selten ist.

Es bliebe noch übrig, von dem Zusammenhang der Infektionskrankheiten sowie der Magen- und Darmleiden mit den sich ihnen anschliessenden Tetanien zu sprechen. Positive Kenntnisse bezüglich dieser Fälle besitzen wir nicht.

Bezüglich der ersteren könnten wir daran denken, dass in einigen Fällen die Tetanie thyreoidalen Ursprungs wäre, nachdem bei ein und der anderen akuten Infektionskrankheit manchmal Schilddrüsenentzündung beobachtet wurde. Ich halte es jedoch für wahrscheinlicher, dass hier Produkte von das betreffende Leiden verursachenden Mikroorganismen als Krankheitserreger fungieren; und zur Unterstützug dieser Ansicht möchte ich anführen, dass wir die von seiten des Gehirns in akuten Infektionskrankheiten sich einstellenden Symptome ebenfalls diesen Toxinen zuschreiben und so könnte man sich vorstellen, dass dieselben auch im Rückenmark solche Veränderungen zustande bringen, die sich in Reizerscheinungen äussern und mit dem Verschwinden des Leidens ebenfalls aufhören.

Bezüglich der sich an Magenleiden anschliessenden Tetanien ist 
die am allgemeinsten angenommene Hypothese ebenfalls die Autointoxikationstheorie.

Wenn wir nun im Folgenden unsere auf die Ätiologie der Tetanie Erwachsener bezüglichen positiven Kenntnisse sowie Hypothesen zusammenfassen, so können wir dies am einheitlichsten in Folgendem tun. Die Tetanie, welche sich in einer auf meehanische und elektrische Einwirkungen gesteigerten Reizbarkeit des Nervensystems äussert, ist die Folge einer durch Gifte verursachten Ernährungsstörung des Nervensystems. Die Gifte, welche eine solche Veränderung des Nervensystems hervorrufen, können zweifachen Ursprungs sein: entweder bilden sie sich im Organismus, wie bei der Tetania strumipriva und in den sich zur Gravidität und Laktation, zu Infektionskrankheiten sowie zu Magen- und Darmleiden gesellenden Fällen, oder aber sie kommen von aussen in den Organismus, wie bei den in Verbindung mit akuten und chronischen Vergiftungen auftretenden Tetanien, sowie in den Fällen idiopathischer Tetanie, bezüglich welcher ich, wie ich dargelegt habe, ebenfalls den Verdacht hege, dass irgend ein von aussen eingedrungenes Gift das Nervensystem angreife, d. h. dass wir es mit einer gewerblichen, manchmal vielleicht mit einer technischen oder härslichen Vergiftung zu tun haben. Bezüglich der von aussen eingeführten Gifte glaube ich, dass am häufigsten das Blei als Krankheitsursache der Tetanie figuriert, doch muss ich ausserdem auf Grund meiner vorausgeschickten $\mathrm{Ab}$ leitungen es für wahrscheinlich halten, dass auch Quecksilber, Phosphor, Arsen, Kupfer (Kétly ${ }^{1}$ ) sah in Verbindung mit einer solchen Vergiftung 'Tetaniesymptome) sowie andere auf das Nervensystem schädlich wirkende Stoffe das Leiden hervorrufen können.

Ob nun all diese organischen und unorganischen Gifte, welche ich im Vorstehenden verdächtigt habe, unmittelbar das Nervensystem angreifen oder vielleicht in Blute oder in den die Zellen ernährenden Schlagadern (Picks pathologisch-histologische Befunde) krankhafte Veränderungen zustande bringen und die Ernährungsstörung der Nervenzellen auf dieser Grundlage zustande kommt, ist eive Frage, auf die wir noch die Antwort schuldig sind; doch halte ich meinerseits das Erstere für wahrscheinlicher.

Das hier Zusammengefasste würde nach dem heutigen Stande unsereres Wissens am besten jene zahllosen Eigentümlichkeiten er1) A belgyógyászat kézikönyve. 2. Bd. Praktisch wichtigere Vergiftungen.
Kupfer. 1) A belgyógyáásat kézikönyve. 2. Bd. Praktisch wichtigere Vergiftungen.
Kupfer. 
klären, die wir im Auftreten der Tetanie sehen, und würde auch jene Erfahrung verstehen machen, dass die bei infektiösen Leiden sowie im Verlaufe akuter Vergiftungen auftretenden Tetanien bloss einige Tage dauern; es würde begreiflich machen, warum die Maternitätstetanien am ehesten während einer neuen Mutterschaft rezidivieren, und warum. in den bei Handwerkern vorkommenden Fällen sich das Leiden oft Jahre hindurch zeigt.

Wenn ich nun auf die Frage zurückkomme, warum die Tetanie in den Winter- und Frühjahrsmonaten am häufigsten ist, so könnte ich vielleicht auch die Beleuchtung des "rheumatischer Einfluss“ genannten, unbekannten Erregers mit einigen Bemerkungen versuchen. Ob nicht vielleicht die Ausmerzung der früher erwähnten Gifte leichter im Sommer erfolgt, resp. im Winter erschwert ist, durch die verminderte Hauttransfusion und Schweissabsonderung, durch die mangelhaftere Pflege der Haut, sowie durch die infolge der Kälte eintretende Zusammenziehung der Blatgefässe der Oberdecke.

Ein weiteres Forschen nach der Atiologie der Tetanie in der Richtung der hier entwickelten Hypothesen ist meiner Ansicht nach der Mühe wert. 